\title{
The stability of the equilibrium of two-phase elastic solids
}

\author{
V.A. Yeremeyev, A.B. Freidin, L.L. Sharipova \\ Rostov-on-Don, St. Petersburg, Russia
}

\begin{abstract}
The distinctive features of the loss of stability of elastic solids which undergo phase transitions are investigated for the case of small deformations. The non-uniqueness of the solution of the boundary-value problem for the describing of the thermodynamic equilibrium of a two-phase body is caused by the non-linearity associated with the unknown interface. The solution can be chosen by comparing the potential energies of the body in the two-phase and single phase states and by analysing of the local stability of the two-phase states. A linearized boundary-value problem is formulated which describes infinitesimal small perturbations of an initial two-phase state which is in thermodynamic equilibrium. Analysis of the stability of the two-phase state reduces to an investigation of the bifurcation points and the behaviour of the small solutions of the system of integrodifferential equations in terms of functions describing the perturbations of the interface. The problem of the non-uniqueness and loss of stability of centrisymmetric equilibrium two-phase deformations is investigated as an example. A theorem concerning the number of centrisymmetric solutions is proved. The energy changes accompanying the formation and development of two-phase states and the stability of the solutions obtained are investigated. The concept of topological instability as a bifurcation is introduced, as a result of which the type of geometry of a solution of the boundary-value problem changes and surfaces of separation of the phases actually appear and disappear. Macrodiagrams of the deformational are constructed which demonstrate the effect of deformation softening in the path of a phase transition.
\end{abstract}

Investigations of phase transitions during the deformation of solids lie at the interface of material science, solid state physics and continuum mechanics and, in practice, are orientated towards the use and development of materials which react to external actions in a specified and non-trivial manner, including shape memory materials. ${ }^{1,2}$ The distinctive features of the rheological behaviour of a material, the localization of the deformations and heterogeneous deformation can also be the result of structural transformations as, for example, in the case of martensite plasticity in metals or orientational transitions in polymers.

Phase transitions with a clear interface are considered below. Such interfaces arise during martensite transitions. ${ }^{3}$ The approach developed here goes back to the work of $\mathrm{Gibbs}^{4}$ on the phase equilibria of liquids and gases. The interface in deformed material is considered as a surface of discontinuity in the deformations in the case of a continuous displacement field, and an additional condition of thermodynamic equilibrium is postulated on this surface (an analogue of the equality of the chemical potentials in Gibbs' theory) which is necessary in order to determine it. ${ }^{5-27}$ 
Note that, in addition to this approach, theories are being developed at the present time which are based on the introduction of additional parameters of state, which characterize features of the structure of the material "in the mean" (for example, the concentration of a new phase) and the formulation of relations for them ${ }^{28-30}$ (see also the bibliography in Ref. 2). While enabling the important features of the deformation processes associated with phase transitions to be revealed, these theories omit the stage in which the interface is explicitly considered which, on the one hand, avoids the need to solve problems with unknown boundaries but, on the other hand, excludes the detailed stress and strain fields at the interface from consideration and the two-phase microstructure proper, which, generally speaking, arises and develops in a different manner along the different deformation paths.

Being problems with an unknown internal boundary, problems of the equilibrium two-phase configurations of elastic bodies have, as a rule, a non-unique solution (see, for example, Refs. 18-20). The solution can be chosen by estimating the energy changes occurring in the body as a consequence of the phase transitions and analysing the stability of the two-phase states. ${ }^{31,32}$ An investigation of the stability of two-phase states is also an important technological problem. For example, loss of stability during crystal growth can be accompanied by the appearance of corrugations on the surface of the sample or the growth of dendrites. ${ }^{33-35}$ Instability accompanying the solidification of castings can lead to bulging of the surface of a hardening casting and the appearance of cracks, caverns and other defects. ${ }^{36}$ On the other hand, loss of stability of the thin-walled elements of structures made of materials which undergo phase transitions can be used in the design of transducers and micromotors. ${ }^{2}$

Various problems associated with the stability of deformable bodies when there are phase transitions have been considered earlier in Refs. 15,31,32,37-46. We also note investigations of morphological stability within the framework of the Stefan problem. ${ }^{34,35}$

In this paper, a general procedure is developed for investigating the stability of the two-phase states of elastic solids in the case of small deformations, which is based on an analysis of the evolution of perturbations of the equilibrium interface. Only the effect of the relation between the deformation and phase transitions processes is discussed. The possibility of accompanying processes, such as fracture or plasticity caused by a phase transitions as well as competition between the different processes are not considered.

The destabilizing role of phase transitions is investigated using the example of centrisymmetric deformations. The equilibrium of a two-phase sphere in a class of solutions with a single spherical interface was considered previously in Refs. 18-20,23,37 It has been shown that: 1) two solutions are possible for one and the same boundary conditions: when the domain of the new phase develops from the centre or from the surface of the sphere; 2) if radial displacements are specified on the sphere surface, then both solutions are stable in the class of centrisymmetric solutions, when only the radius of the interface is perturbed; both solutions are unstable if a pressure is specified on the sphere surface (the latter implies that the boundary conditions in the strains and the stresses are not equivalent); 3) both two-phase states, if they exist, are thermodynamically preferred if a displacement is specified on the surface; if a pressure is specified on the surface, then the potential energy of a two-phase state is greater than that of a single phase state; 4) competition between the development of the domain of a new phase from the centre of the sphere or from its surface is determined by the ratio of the shear moduli of the phases; a solution, for which the phase with the greater shear modulus is located at the centre, corresponds to a lower energy of the two-phase state.

These investigations leave open questions of the possibility of two or more equilibrium interfaces and the stability of the two-phase states to arbitrary perturbations. These questions were partially considered earlier in Refs. 31,32,40. It is shown below that the additional thermodynamic conditions does, in fact, constrain the number of equilibrium solutions. The stability of all possible equilibrium centrisymmetric two-phase deformations is subsequently investigated. The strain fields, corresponding to the different centrisymmetric two-phase states of the sphere, are compared with the zone of phase transitions which is formed in deformation space by all deformations which can exist in a given material in the equilibrium boundaries of the phases. ${ }^{16,19,21}$ It is shown that the number of centrisymmetric two-phase solutions can be predicted a priori, if the zone of the phase transitions is constructed. Different strain fields on the sphere correspond to different solutions to which, in their turn, different curves in the strain space correspond. How the curves corresponding to unstable solutions are related to the phase transition zone is investigated.

\section{The equilibrium of two-phase elastic solids in the case of small deformations}

In the case of small deformations, the problem of the equilibrium two-phase configurations of an elastic soild during diffusionless phase transitions can be reduced to the problem of determining the interface $\Gamma$ and the corresponding strain 


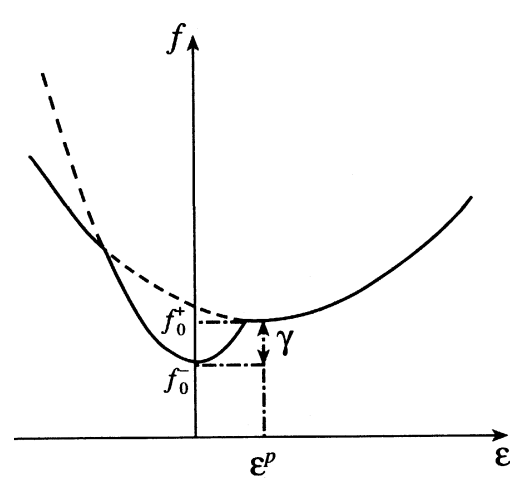

Fig. 1 .

field $\mathbf{u}(\mathbf{x})$, which is fairly smooth when $\mathbf{x} \notin \Gamma$, continuous on $\Gamma$ and satisfies the equilibrium and boundary conditions (see Refs. 13,14,20,21

$$
\begin{aligned}
& \mathbf{x} \notin \Gamma: \nabla \cdot \boldsymbol{\sigma}=0, \quad \theta=\text { const } \\
& \mathbf{x} \in \Gamma:[\mathbf{u}]=0, \quad[\boldsymbol{\sigma}] \cdot \mathbf{n}=0,[f]-\boldsymbol{\sigma}:[\boldsymbol{\varepsilon}]=0
\end{aligned}
$$

where $\boldsymbol{\sigma}=\partial f / \partial \boldsymbol{\varepsilon}$ is the stress tensor, $\mathbf{n}$ is a unit vector of the normal to the interface $\Gamma, \mathbf{u}$ is the displacement vector, $\boldsymbol{\varepsilon}$ is the strain tensor and $\theta$ is the temperature; the bulk free energy density $f$ is modelled by the quadratic dependences (Fig. 1)

$$
\begin{aligned}
& f(\boldsymbol{\varepsilon}, \boldsymbol{\theta})=\min _{-,+}\left\{f^{-}(\boldsymbol{\varepsilon}, \boldsymbol{\theta}), f^{+}(\boldsymbol{\varepsilon}, \boldsymbol{\theta})\right\} \\
& f^{ \pm}(\boldsymbol{\varepsilon}, \boldsymbol{\theta})=f_{0}^{ \pm}(\boldsymbol{\theta})+\frac{1}{2}\left(\boldsymbol{\varepsilon}-\boldsymbol{\varepsilon}_{ \pm}^{p}\right): \mathbf{C}_{ \pm}:\left(\boldsymbol{\varepsilon}-\boldsymbol{\varepsilon}_{ \pm}^{p}\right)
\end{aligned}
$$

The plus and minus signs refer to the material in different phase states, and, henceforth, correspondence of the subscripts and superscripts and the plus and minus signs in the equalities is understood, the minus and plus phases occupy the domains $V_{-}$and $V_{+}$respectively, the change in a quantity on transferring from the minus phase to the plus phase is denoted by square brackets, $\mathbf{C}_{ \pm}$are the positive definite tensors of the moduli of elasticity of the phases, and the parameters $f_{0}^{ \pm}$and $\boldsymbol{\varepsilon}_{+}^{p}$ have the meaning of the free energy densities and strain tensors of the phases in the unstressed state (if one puts $\boldsymbol{\varepsilon}_{-}^{p}=0$, then $\left[\boldsymbol{\varepsilon}^{p}\right]=\boldsymbol{\varepsilon}^{p}$ is the strain tensor in the case of a hypothetical phase transition from one homogeneous stress-free state into another, that is, the characteristic deformation of the transition). Mass forces, thermoelastic stresses and the surface energy are not taken into account. If $\mathbf{C}_{ \pm}$and $\boldsymbol{\varepsilon}_{ \pm}^{p}$ are independent of temperature, then the parameter $\gamma(\theta)=\left[f_{0}\right]$ plays the role of a temperature. If the "temperature" $\gamma$ is such that the paraboloids (1.4) do not intersect, then two-phase equilibrium states are impossible. ${ }^{21}$

Equation (1.1) and the first two equations at the discontinuity (1.2) are the usual equations in the case of the equilibrium of a composite solid. The third of conditions (1.2) is an additional condition of thermodynamic equilibrium associated with the additional degree of freedom generated by the unknown interface and follows from the conditions for the equilibrium of phases of a non-linear elastic body, which have been discussed earlier (Refs. 5-9,12,15,22,25. Since, by virtue of the continuity of the displacements, we can represent the discontinuity in the strains in the form of a symmetrized dyadic

$$
[\boldsymbol{\varepsilon}]=1 / 2(\mathbf{n} \otimes \mathbf{a}+\mathbf{a} \otimes \mathbf{n})
$$

(a is the vector of the amplitude of the discontinuity), then, by virtue of the continuity of the forces (of the second of conditions (1.2)), the stress tensor in the third of conditions (1.2) can be taken on any of the sides of the interface.

The constitutive relations in the linear theory of elasticity of an inhomogeneous medium

$$
\boldsymbol{\sigma}_{ \pm}(\varepsilon)=\mathbf{C}_{ \pm}:\left(\varepsilon-\varepsilon_{ \pm}^{p}\right), \quad \varepsilon \in \mathscr{E}_{ \pm}
$$


where, according to relations (1.3) and (1.4), the domains of definition of the phases in deformation space are:

$$
\mathscr{E}_{-}=\{\boldsymbol{\varepsilon}: \varphi(\boldsymbol{\varepsilon})>0\}, \quad \mathscr{E}_{+}=\{\boldsymbol{\varepsilon}: \varphi(\boldsymbol{\varepsilon})<0\}, \quad \varphi(\boldsymbol{\varepsilon})=f^{+}(\boldsymbol{\varepsilon})-f^{-}(\boldsymbol{\varepsilon})
$$

correspond to relations (1.3).

The condition for the continuity of the force, taking account of representation (1.5), is rewritten in the form of a formula for determining of the discontinuity in the deformations (or stresses) as a function of the normal and the deformations (or stresses) on one of the sides of the interface ${ }^{21,47}$

$$
\begin{aligned}
& {[\boldsymbol{\varepsilon}]=\mathbf{K}_{\mp}(\mathbf{n}): \mathbf{q}_{ \pm}} \\
& \mathbf{q}_{ \pm}=-\mathbf{C}_{1}: \boldsymbol{\varepsilon}_{ \pm}+\left[\mathbf{C}: \boldsymbol{\varepsilon}^{p}\right], \quad \mathbf{C}_{1}=\mathbf{C}_{+}-\mathbf{C}_{-} \\
& \mathbf{K}_{ \pm}(\mathbf{n})=\left\{\mathbf{n} \otimes \mathbf{G}_{ \pm}(\mathbf{n}) \otimes \mathbf{n}\right\}^{s}, \quad \mathbf{G}_{ \pm}(\mathbf{n})=\left(\mathbf{n} \cdot \mathbf{C}_{ \pm} \cdot \mathbf{n}\right)^{-1}
\end{aligned}
$$

The superscript $s$ denotes the operation of symmetrization with respect to the subcripts within the first or second pair: $K_{i j k l}=n_{(i} G_{j)(k} n_{l)}$.

When account is taken of relations (1.3) and (1.7), the thermodynamic condition is reduced to the form ${ }^{13,21}$

$$
\chi\left(\boldsymbol{\varepsilon}_{ \pm}, \mathbf{n}\right) \triangleq \gamma+\frac{1}{2}\left[\boldsymbol{\varepsilon}^{p}: \mathbf{C}: \boldsymbol{\varepsilon}^{p}\right]+\frac{1}{2} \boldsymbol{\varepsilon}_{ \pm}: \mathbf{C}_{1}: \boldsymbol{\varepsilon}_{ \pm}-\boldsymbol{\varepsilon}_{ \pm}:\left[\mathbf{C}: \boldsymbol{\varepsilon}^{p}\right] \pm \frac{1}{2} \mathbf{q}_{ \pm}: \mathbf{K}_{\mp}(\mathbf{n}): \mathbf{q}_{ \pm}=0
$$

Hence, the system of conditions at the interface (1.2) is decomposed. For a given value of $\gamma$, any of Eqs. (1.9) defines a single-parameter family of unit normals as a function of the deformations at one of the sides of the interface (that is, the plus or minus phase) The deformations at the other side of the interface are calculated from formulae (1.7), and the second of the Eq. (1.9) is automatically satisfied.

If the tensor $\mathbf{C}_{1}$ is non-degenerate, then the thermodynamic condition (1.9) can be written in $q$-space ${ }^{13,21}$

$$
\begin{aligned}
& \chi\left(\mathbf{q}_{ \pm}, \mathbf{n}\right) \triangleq \gamma_{*}+\frac{1}{2} \mathbf{q}_{ \pm}:\left(\mathbf{C}_{1}^{-1} \pm \mathbf{K}_{\mp}(\mathbf{n})\right): \mathbf{q}_{ \pm}=0 \\
& \gamma_{*}=\gamma+\frac{1}{2}\left[\boldsymbol{\varepsilon}^{p}\right]: \mathbf{B}_{1}^{-1}:\left[\boldsymbol{\varepsilon}^{p}\right], \quad \mathbf{B}_{ \pm}=\mathbf{C}_{ \pm}^{-1}, \quad \mathbf{B}_{1}=\mathbf{B}_{+}-\mathbf{B}_{-}
\end{aligned}
$$

In the case of quasistatic motion of the interface, condition (1.7) remains valid and the quantity $\chi \neq 0$ is the thermodynamic force, since the energy dissipation $D$ during the quasistatic propagation of the surface of discontinuity in the deformations can be represented in the form

$$
D=-\int_{\Gamma} \chi v_{n}^{\Gamma} d \Gamma
$$

where $v_{n}^{\Gamma}$ is the velocity of motion of the surface $\Gamma$ in the direction of the normal (this normal is the outward normal to the domain occupied by the plus phase). This follows from a general analysis of the change in the potential energy of a body accompanying the quasistatic motion of an interface in a linear elastic body. ${ }^{48}$ In the approximation of the linear thermodynamics of irreversible processes, the normal component of the velocity of the interface is determined by the kinetic equation

$$
v_{n}^{\Gamma}=-k \chi\left(\mathbf{q}_{ \pm}, \mathbf{n}\right), \quad k>0
$$

where $k$ is a kinetic coefficient. Note that

$$
\chi\left(\boldsymbol{\varepsilon}_{-}, \mathbf{n}\right)=\chi\left(\boldsymbol{\varepsilon}_{+}, \mathbf{n}\right)=\chi\left(\mathbf{q}_{-}, \mathbf{n}\right)=\chi\left(\mathbf{q}_{+}, \mathbf{n}\right)
$$

Actually, from the definition of the function $\chi\left(\boldsymbol{\varepsilon}_{ \pm}, \mathbf{n}\right)$ and condition (1.7), it follows that

$$
\begin{aligned}
& \chi\left(\boldsymbol{\varepsilon}_{+}, \mathbf{n}\right)-\chi\left(\boldsymbol{\varepsilon}_{-}, \mathbf{n}\right)=\frac{1}{2}\left[\boldsymbol{\varepsilon}: \mathbf{C}_{1}: \boldsymbol{\varepsilon}\right]-[\boldsymbol{\varepsilon}]:\left(\left[\mathbf{C}: \boldsymbol{\varepsilon}^{p}\right]-\frac{1}{2}\left(\mathbf{q}_{+}+\mathbf{q}_{-}\right)\right)= \\
& =\frac{1}{2}\left[\boldsymbol{\varepsilon}: \mathbf{C}_{1}: \boldsymbol{\varepsilon}\right]-\frac{1}{2}[\boldsymbol{\varepsilon}]: \mathbf{C}_{1}:\left(\boldsymbol{\varepsilon}_{+}+\boldsymbol{\varepsilon}_{-}\right) \equiv 0
\end{aligned}
$$

Relation (1.11) will be used later to analyse of the evolution of perturbations. 


\section{The linearized boundary-value problem for a two-phase body}

The investigation of the stability of an equilibrium state involves a consideration of equilibrium positions which are slightly different from that which has been found and determining the values of the loading parameters for which the existence of non-trivial solutions of the perturbed problem is possible. The determination of the bifurcation points of the solutions of the perturbed problem is supplemented with an analysis of the evolution of the perturbation of the interface which provides information on the behaviour of the solution in the neighbourhood ("before" and "after") the bifurcation points.

The displacements of the points of the body and the radius vector of the interface in the perturbed state are given by the formulae

$$
\mathbf{u}=\mathbf{u}^{0}+\mathbf{w}, \quad \mathbf{r}=\mathbf{r}^{0}+\eta \mathbf{n}
$$

where $\mathbf{u}^{0}$ and $\mathbf{r}^{0}$ are the displacement vectors and the position of the interface in the unperturbed (initial) state, $\mathbf{w}$ is the vector of the small perturbations of the displacements and $\eta$ is the perturbation of the interface. Quasistatic perturbations are considered. This means that small additional displacements $\mathbf{w}$ are stable at any instant of time, that is, they satisfy the linearized equations of mechanical equilibrium and the boundary conditions, including the condition that the forces at the interface are continuous. On the other hand, the perturbed position of the interface, which is described using the function $\eta$, does not satisfy the condition of thermodynamic equilibrium, which leads to the need to study the linearized kinetic equation (1.11). From a physical point of view, the assumption that the process is quasistatic means that the characteristic times for the establishment of mechanical equilibrium are far shorter than the time taken to reach thermodynamic equilibrium.

Linearization of the boundary-value problem for a two-phase body in the case of small deformations leads to a system of equations and boundary conditions in $\mathbf{w}$ and $\eta$. The relations

$$
\begin{aligned}
& \mathbf{x} \notin \Gamma: \nabla \cdot \boldsymbol{\sigma}_{ \pm}(\mathbf{w})=0, \quad \boldsymbol{\sigma}_{ \pm}(\mathbf{w})=\mathbf{C}_{ \pm}: \nabla \mathbf{w} \\
& \mathbf{x} \in \Omega_{1} \cup \Omega_{2}:\left.\mathbf{w}\right|_{\Omega_{1}}=0,\left.\quad \mathbf{n} \cdot \boldsymbol{\sigma}(\mathbf{w})\right|_{\Omega_{2}}=0 \\
& \left.\boldsymbol{\sigma}(\mathbf{w})\right|_{\Omega_{2}}= \begin{cases}\boldsymbol{\sigma}_{-}(\mathbf{w}), & \left.\mathbf{x}\right|_{\Omega_{2}} \in V_{-} \\
\boldsymbol{\sigma}_{+}(\mathbf{w}), & \left.\mathbf{x}\right|_{\Omega_{2}} \in V_{+}\end{cases}
\end{aligned}
$$

must be satisfied in the domains $V_{ \pm}$occupied by the different phases and at the boundary of the body, and the relations

$$
\begin{aligned}
& {[\mathbf{w}]=-\eta\left[\mathbf{n} \cdot \nabla \mathbf{u}^{0}\right]} \\
& \mathbf{n} \cdot[\boldsymbol{\sigma}(\mathbf{w})]=\nabla \eta \cdot\left[\boldsymbol{\sigma}^{0}\right]-\eta \mathbf{n} \cdot\left[\mathbf{n} \cdot \nabla \boldsymbol{\sigma}^{0}\right] \\
& \mathbf{q}_{ \pm}^{0}: \boldsymbol{\varepsilon}\left(\mathbf{w}_{ \pm}\right) \mp \mathbf{q}_{ \pm}^{0}: \mathbf{K}_{\mp}(\mathbf{n}): \mathbf{q}\left(\mathbf{w}_{ \pm}\right)-\eta \mathbf{n} \cdot \nabla \chi\left(\boldsymbol{\varepsilon}\left(\mathbf{u}_{ \pm}^{0}\right), \mathbf{n}\right)=\frac{1}{k} \frac{d \eta}{d t}
\end{aligned}
$$

must be satisfied at the interface $\Gamma$, where $\boldsymbol{\sigma}_{ \pm}^{0}=\mathbf{C}_{ \pm}:\left(\boldsymbol{\varepsilon}\left(\mathbf{u}_{ \pm}^{0}\right)-\boldsymbol{\varepsilon}_{ \pm}^{0}\right)$ and $\mathbf{q}_{ \pm}^{0}=-\mathbf{C}_{1}: \boldsymbol{\varepsilon}\left(\mathbf{u}_{ \pm}^{0}\right)+\left[\mathbf{C}: \boldsymbol{\varepsilon}^{p}\right]$ are the stress tensor and the tensor $\mathbf{q}$ in the unperturbed state, $\boldsymbol{\varepsilon}(\mathbf{u}) \Delta\left(\nabla \mathbf{u}+\nabla \mathbf{u}^{T}\right) / 2, \mathbf{q}\left(\mathbf{w}_{ \pm}\right) \Delta-\mathbf{C}_{1}: \mathbf{\varepsilon}\left(\mathbf{w}_{ \pm}\right)$is the perturbation of the tensor $\mathbf{q}$ and $\Omega_{1} \cup \Omega_{2}$ is the boundary of the body. Conditions (2.3) and (2.4) follow from the continuity of the displacements and forces at the unperturbed interface respectively, and Eq. (2.5) follows from relations (1.9) and (1.11). If the tensor $\mathbf{C}_{1}$ is non-degenerate, then, according to definition (1.10), the linearized kinetic equation (2.5) takes the form

$$
\mathbf{q}_{ \pm}^{0}:\left(\mathbf{C}_{1}^{-1} \pm \mathbf{K}_{\mp}(\mathbf{n})\right): \mathbf{q}\left(\mathbf{w}_{ \pm}\right)+\eta \mathbf{n} \nabla \chi\left(\mathbf{q}_{ \pm}, \mathbf{n}\right)=-\frac{1}{k} \frac{d \eta}{d t}
$$

Either of the two relations (2.5) (with the upper or lower signs and plus/minus subscripts) or (2.6) describes the quasistatic evolution of the perturbed interface as a function of the initial state and the perturbation of the displacements at one of the sides of the interface. If the initial state contains several interfaces, conditions (2.3)-(2.5) are set up on each of them. 
When there is no phase transition $\eta \equiv 0$. The homogeneous boundary-value problem (2.1)-(2.4) describes the deformation of a composite linear elastic solid (a composite) and only admits of trivial solutions (null solutions or solutions corresponding to the motion of an absolutely rigid body).

If perturbations of the interface are allowed $(\eta \neq 0)$, then, since the operator, which is generated by boundaryvalue problem (2.1)-(2.4) (without reference to the thermodynamic condition) is uniquely solvable, the additional displacements $\mathbf{w}_{ \pm}$can be expressed in terms of $\eta$ :

$$
\mathbf{w}_{ \pm}=\mathscr{A}_{ \pm} \eta
$$

where $\mathscr{A}_{ \pm}$are certain linear integral operators. By virtue of the connectedness of the problem of determining the displacement field and the positions of the interface, the perturbations $\mathbf{w}_{ \pm}$depend on $\eta$ and, consequently, the dependence of $\eta$ on $t$ also implies that $\mathbf{w}_{ \pm}$depends on $t$.

Substitution of expressions (2.7) into kinetic equation (2.6) leads to an integrodifferential equation for the function $\eta$

$$
-\frac{1}{k} \frac{d \eta}{d t}=\mathscr{L} \eta
$$

where $\mathscr{L}$ is a linear integrodifferential operator which is defined by the equality

$$
\mathscr{L} \eta=-\mathbf{q}_{ \pm}^{0}:\left(\mathbf{C}_{1}^{-1}+\mathbf{K}_{-}(\mathbf{n})\right): \mathbf{C}_{1}: \boldsymbol{\varepsilon}\left(\mathbf{w}_{ \pm}\right)+\eta \mathbf{n} \cdot \nabla \chi\left(\mathbf{q}_{ \pm}^{0}, \mathbf{n}\right)
$$

In the case of several interface $\Gamma_{1}, \Gamma_{2}, \ldots \Gamma_{m}$, the solution of Eqs. (2.1)-(2.5) (or (2.1)-(2.4), (2.6)) leads to a system of integrodifferential equations for the perturbations of the interfaces $\eta_{j}$

$$
-\frac{1}{k_{i}} \frac{d \eta_{i}}{d t}=\mathscr{L}_{i j} \eta_{j}, \quad i, j=1,2, \ldots, m
$$

where $k_{i}$ and $\mathscr{L}_{i}$ are kinetic coefficients and integrodifferential operators, corresponding to the interfaces $\Gamma_{i}$.

Hence, in the case of small perturbations of a two-phase solid, a loss of stability of the solutions of boundaryvalue problem (1.1), (1.2) is solely associated with the existence of an additional degree of freedom (the position of the interface) and the thermodynamic equation corresponding to it. Investigation of the stability of the equilibrium solutions leads to the determination of the bifurcation points of system (2.9) and the behaviour of its small solutions.

If the operator $\mathscr{L}=\left\{\mathscr{L}_{i j}\right\}$ is positive definite, then system (2.9) only admits of time-decreasing solutions. In this case, the initial two-phase state is stable. Otherwise, the perturbations do not decrease and the solution is unstable. The bifurcation points are determined from the condition for the existence of non-zero solutions of the equations

$$
\mathscr{L}_{i j} \eta_{j}=0, \quad i, j=1,2, \ldots, m
$$

Note that, in the case of finite deformations, loss of stability is possible for reasons other than phase transitions. This leads to competition between the causes of loss of stability in non-linear elastic bodies undergoing phase transitions (see, for example, Refs. 37 and 46). The analysis proposed below, taking the problem of a two-phase sphere as an example, enables us to separate out the effect of just the phase transition on stability.

\section{The non-uniqueness of equilibrium centisymmetric two-phase strains}

We will investigate the possibility of the existence of centisymmetric equilibrium two-phase states with several spherical interfaces dividing a body in the domain

$$
V_{i}=\left\{\varphi \in[0,2 \pi], \theta \in[0, \pi], r_{i-1}<r \leq r_{i}\right\}, \quad i=1,2, \ldots, N+1
$$

where $r, \varphi$ and $\theta$ are spherical coordinates, $r_{i}(i=1,2, \ldots, N)$ are the radii of the interfaces, $r_{N+1}=R$ in the case of a sphere of radius $R$ and $r_{N+1} \rightarrow \infty$ in the case of an unbounded medium. Moreover, a cavity, bounded by a surface $r=r_{0}(\varphi, \theta)$, for which boundary conditions permitting centrisymmetric deformations are specified, is allowed to exist in the central part of the body. In the case of a spherical cavity with its centre coinciding with the centre of the spherical interfaces, a radial displacement or pressure $r_{0}=$ const can be specified on the surface. 
The relations

$$
r=r_{i}:[u]=0, \quad\left[\sigma_{r}\right]=0, \quad i=1, \ldots, N
$$

are satisfied regardless of the thermodynamic condition at the interface, where $u=u(r)=u_{\Gamma} u_{\theta}=u_{\varphi}=0$ are the components of the displacement vector in a polar system of coordinates. In the case of a solid, the displacements at the centre of the sphere are bounded:

$$
\left.u\right|_{r \rightarrow 0}<\infty
$$

The radial displacements in the domains $V_{i}$ are given by Lamé's formulae

$$
u_{i}=A_{i} r+D_{i} / r^{2}, \quad i=1, \ldots, N+2
$$

For specified boundary conditions on the outer surface of the body or at infinity (in the case of an inhomogeneous medium), the $2 N+2$ dependences of $A_{\mathrm{i}}$ and $D_{\mathrm{i}}$ on $r_{i}, r_{2}, \ldots r_{N}$ are determined by conditions (3.1) and (3.2) (or by the condition on the cavity surface) for any number of interfaces. An analysis of the possibility of satisfying condition (1.10) on all the interfaces enables us to formulate the following assertion.

Theorem. If the material is isotropic, then, in the class of centrisymmetric equilibrium two-phase solutions, only a single spherical interface can exist in the case of a solid and no more than two interfaces in the case of a body with a cavity at the centre.

Proof. For an isotropic material

$$
\begin{aligned}
& \mathbf{C}_{ \pm}=\lambda_{ \pm} \mathbf{E} \otimes \mathbf{E}+2 \mu_{ \pm} \mathbf{I}, \quad \boldsymbol{\varepsilon}_{+}^{p}=\left(\vartheta^{p} / 3\right) \mathbf{E}, \quad \boldsymbol{\varepsilon}_{-}^{p}=0 \\
& \mathbf{K}^{ \pm}(\mathbf{n})=\frac{1}{\mu_{ \pm}}(\mathbf{n} \otimes \mathbf{E} \otimes \mathbf{n})^{s}-\frac{1}{2 \mu_{ \pm}\left(1-v_{ \pm}\right)} \mathbf{n} \otimes \mathbf{n} \otimes \mathbf{n} \otimes \mathbf{n} \\
& \boldsymbol{\sigma}_{ \pm}=\left(\lambda_{ \pm} \operatorname{tr} \boldsymbol{\varepsilon}_{ \pm}-K_{ \pm} \vartheta_{ \pm}^{p}\right) \mathbf{E}+2 \mu_{ \pm} \boldsymbol{\varepsilon}_{ \pm}, \quad\left(\vartheta_{-}^{p}=0\right)
\end{aligned}
$$

where $\mathbf{E}$ and $\mathbf{I}$ are unit tensors of the second and fourth rank respectively, $\lambda$ and $\mu$ are Lamé coefficients, $\nu$ is Poisson's ratio and $K$ is the of bulk compression modulus.

Without loss of generality, we will assume that the domain $V_{1}$ is occupied by the plus phase, that is, $V_{1}=V_{1}^{+}, V_{2}=$ $V_{2}^{-}, V_{3}=V_{3}^{+}$etc. $\mathbf{C}_{1} \neq 0$. The tensors $\mathbf{q}_{i}^{+}$in the domains $V_{i}$ are axisymmetric when $i \geq 2$ if there is no cavity, and, when $i \geq 1$, if there is a cavity:

$$
\begin{aligned}
& \mathbf{q}_{i}^{+}=-\mathbf{C}_{1}: \boldsymbol{\varepsilon}_{i}^{+}+\mathbf{C}_{+}: \boldsymbol{\varepsilon}^{p}=\left(t-3 K_{1} A_{i}\right) \mathbf{E}-\frac{2 \mu_{1} D_{i}}{r^{3}}(\mathbf{E}-3 \mathbf{n} \otimes \mathbf{n}) \\
& K_{1}=K_{+}-K_{-}, \quad \mu_{1}=\mu_{+}-\mu_{-}, \quad t=K_{+} \vartheta^{p}
\end{aligned}
$$

If the body is solid, the tensor $\mathbf{q}$ is spherical in the internal sphere $V_{1}$ and, consequently, at the interface from the direction of the domain $V_{1}$ :

$$
\mathbf{q}_{1}^{+}=q_{1} \mathbf{E}, \quad q_{1}=K_{+} \vartheta^{p}-3 K_{1} A_{1}
$$

We will assume that $N \geq 2$ for a solid and that $N \geq 3$ when $r_{0} \neq 0$ and show that this leads to a contradiction.

Suppose that, in the first case, interfaces $r_{1}, r_{2}, \ldots$ exist. Writing conditions (3.1) on the interfaces $r_{1}$ and $r_{2}$ and taking account of the fact that, by virtue of conditions (3.2), $D_{1}=0$, we obtain the system of equations

$$
\begin{aligned}
& A_{2}-A_{1}+D_{2} / r_{1}^{3}=0 \\
& A_{3}-A_{2}+\left(D_{3}-D_{2}\right) / r_{2}^{3}=0 \\
& 3 K_{-} A_{2}-3 K_{+} A_{1}+t-4 \mu_{-} D_{2} / r_{1}^{3}=0 \\
& 3 K_{+} A_{3}-3 K_{-} A_{2}-t-4 \mu_{+} D_{3} / r_{2}^{3}+4 \mu_{-} D_{2} / r_{2}^{3}=0
\end{aligned}
$$


It follows from this system that

$$
A_{3}-A_{1}=\frac{4 \mu_{1} D_{3}}{3 d_{-}^{*} r_{2}^{3}}, \quad d_{ \pm}^{*}=K_{\mp}+\frac{4}{3} \mu_{ \pm}
$$

We now write thermodynamic condition (1.10) at the interfaces $r_{1}$ and $r_{2}$ from the side of the plus phase

$$
\begin{aligned}
& r=r_{1}: q_{1}^{2}=-2 \frac{d_{-}}{d_{-}^{*}} K_{1} \gamma_{*} \\
& r=r_{2}: 2 \gamma_{*}+\frac{12 d_{+}^{*} \mu_{1} D_{3}^{2}}{d_{-} r_{2}^{6}}+\frac{8 \mu_{1}\left(t-3 K_{1} A_{3}\right) D_{3}}{d_{-} r_{2}^{3}}+\frac{d_{-}^{*}\left(t-3 K_{1} A_{3}\right)^{2}}{K_{1} d_{-}}=0 \\
& d_{ \pm}=K_{ \pm}+\frac{4}{3} \mu_{ \pm}, \quad \gamma_{*}=\gamma-\frac{K_{+} K_{-}\left(\vartheta^{p}\right)^{2}}{2 K_{1}}
\end{aligned}
$$

It follows from relations (3.6) and (3.9) that the coefficient $A_{1}$ in the case of thermodynamic equilibrium is uniquely defined by the material parameters. ${ }^{18}$ Substituting this relation into equalities (3.8) we obtain the relation between $A_{3}, D_{3}$ and $r_{2}$, by virtue of which the thermodynamic condition (3.10) can only be satisfied when $D_{3} / r_{2}^{3}=0$. It then follows from system (3.7) that $r_{1}=r_{2}$ and/or $D_{2}=0$. This means that the phase state of the material in the domains $V_{1}$, $V_{2}$ and $V_{3}$ is the same. The case when $K_{1}=0$ is treated in a similar manner.

If, in the case of a cavity, the boundary conditions permit centrisymmetric two-phase deformations, then axisymmetric deformations coexist at the interfaces. Suppose that $N \geq 3$. Writing down conditions (3.1) on the boundaries $r_{1}$ and $r_{2}$, we obtain four equations relating the six unknown constants $A_{i}, D_{i}(i=1,2,3)$. In order to arrive at the contradiction, we analyse the feasibility of condition (1.10), written on the three boundaries $r_{1}, r_{2}$ and $r_{3}$ as viewed from the plus phase. As a result, we obtain that the existence of more than two interfaces is impossible.

\section{Remark.}

$1^{\circ}$. The necessary condition for the existence of equilibrium centrisymmetric two-phase deformations in a solid when $K_{1} \neq 0$

$$
\gamma_{*} K_{1}<0
$$

follows from condition (3.9) (Refs. 18 and 20).

$2^{\circ}$. The multiplicity of solutions is associated both with the possibility of one or two interfaces and, also, with the possibility of a different alternation of the phases.

$3^{\circ}$. Here, solutions are discussed which satisfy equilibrium conditions. The stability of two-phase deformations corresponding to different solutions is considered below.

$4^{\circ}$. In the case when there is a cavity, only the requirement that the tensor $\mathbf{q}$ should be axisymmetric in the domains bounded by the spherical interfaces has been used. It has been assumed that the shape of the cavity and the corresponding conditions on its surface ensure that this requirement is satisfied.

$5^{\circ}$. If the thermodynamic condition is excluded, the centrisymmetric strain field can be an equilibrium field for any number of interfaces.

\section{Analysis of the deformation diagrams}

We will consider a sphere of radius $R$ with a spherical cavity at its centre or without such a cavity. Radial displacements $u_{0}$ are specified on the sphere surface and null forces or null displacements are specified on the cavity surface. We construct deformation diagrams of the sphere as a function of the radii of the interfaces for all centrisymmetric solutions (CSS's). It is assumed that the undeformed sphere is in a minus phase state $(\gamma>0)$. 


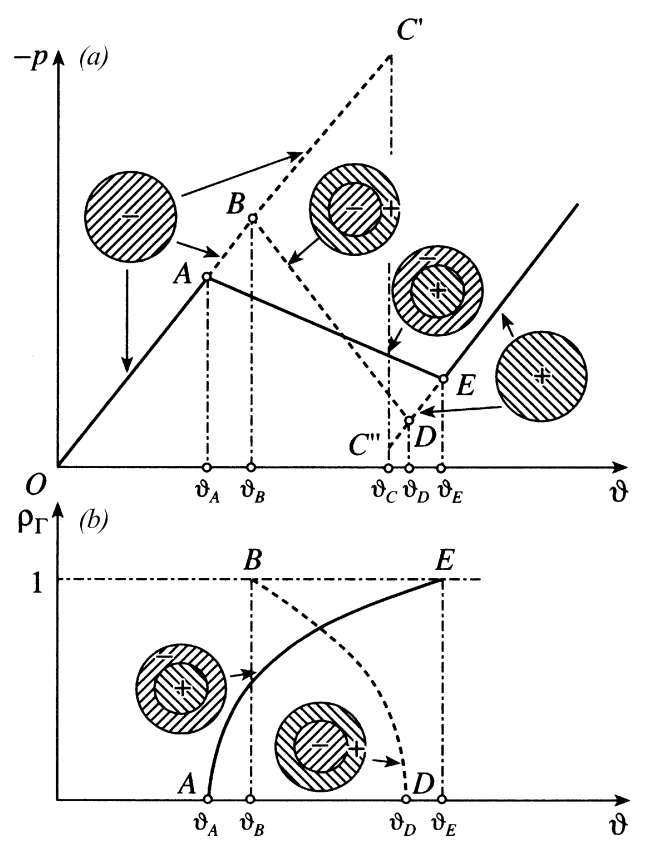

Fig. 2.

\subsection{A solid sphere}

We will now summarize the results obtained for a solid sphere in Refs. 18 and 20. The equilibrium conditions permit two two-phase solutions with a single interface. According to the theorem, there are no other solutions. Typical graphs of the pressure $\rho=-\left.\sigma_{r}\right|_{\mathrm{r}=\mathrm{R}}$ on the sphere surface and the relative radius of the interface $\rho_{\Gamma}=r_{\Gamma} / R\left(r_{\Gamma}\right.$ is the radius of the interface) against the mean bulk deformation $\vartheta=3 u_{0} / R$ are shown in Fig. 2 for the case when $\mu_{+}>\mu_{-}$.

When $0 \leq \vartheta \leq \vartheta_{A}$, the sphere is deformed as a homogeneous linear-elastic body. The solution is represented by the segment $O A$. Other CSS's do not exist. When $\vartheta$ reaches the value $\vartheta_{A}$, a second CSS of boundary-value problem (1.1), (1.2) appears which corresponds to two-phase equilibrium states for which the phase with the greater shear modulus lies at the centre of the sphere (the segment $A E$ ). On reaching $\vartheta_{B}$, yet another CSS (the segment $B D$ ) appears, which corresponds to the development of a new phase from the sphere surface during which the domain within the interface is occupied by the phase with the smaller shear modulus. For both two-phase solutions, the domain of the new phase expands as $\vartheta$ increases and the pressure falls.

\subsection{A sphere with a spherical cavity at the centre}

Suppose $r_{0}$ is the radius of the cavity. According to the theorem, the equilibrium conditions permit the existence of solutions with one or two interfaces and a different alternation of the phases. In the case of two interfaces, the radial displacements in the domains $V_{1}\left(r \in\left[r_{0}, r_{1}\right]\right), V_{2}\left(r \in\left[r_{1}, r_{2}\right]\right)$ and $V_{3}\left(r \in\left(\left[r_{2}, R\right]\right)\right.$ are given by formulae (3.3) when $i=1,2,3$. For each alternation of the phases (plus - minus - plus or minus - plus - minus), when account is taken of relations (3.4) and (3.5), six equations for determining the dependences of the six constants of integration $A_{i}, D_{i}$ on $r_{1}$, $r_{2}$ and $u_{0}$ follow from the boundary conditions

$$
\left.u\right|_{R}=u_{0},\left.\quad \sigma_{r}\right|_{r_{0}}=0\left(\text { or }\left.u\right|_{r_{0}}=0\right), \quad[u]_{r_{\alpha}}=0, \quad\left[\sigma_{r}\right]_{r_{\alpha}}=0 ; \quad \alpha=1,2
$$

The thermodynamic condition (1.10), written for the interfaces $r_{1}$ and $r_{2}$ taking account of relation (3.5), gives a further two equations. As a result, we obtain a system of eight equations in the eight unknowns $A_{i}, D_{i}(i=1,2,3), r_{1}$ and $r_{2}$. The values of $u_{0}$, for which the solution satisfies the conditions $r_{0}<r_{1}<r_{2}<R_{1}$, permits the existence of two-phase states with two interfaces. The number of coefficients $A$ and $D$ and conditions on the interface are correspondingly reduced in the case of just a single interface. 

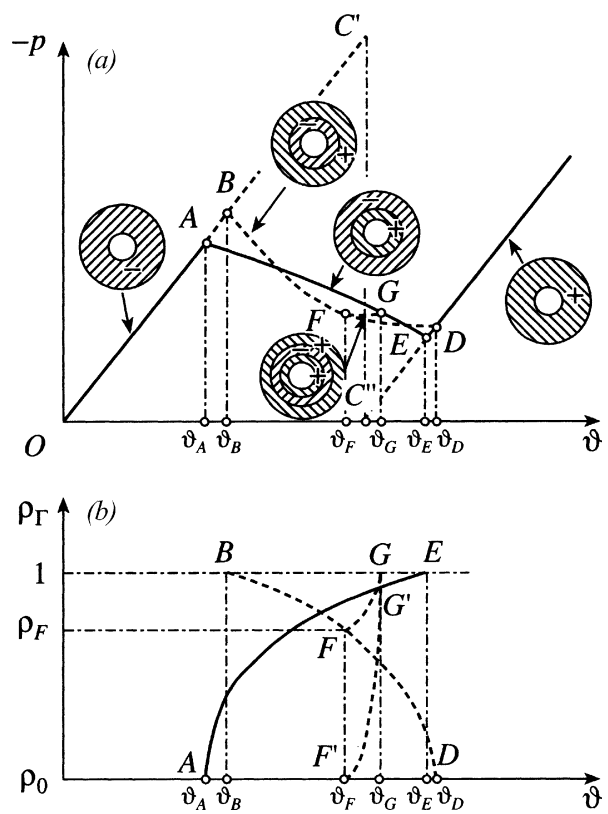

Fig. 3.

Typical diagrams for the deformation of a sphere with a spherical cavity, which undergoes phase transitions according to a mechanism where one or two interfaces are formed, and graphs of the radii of the interfaces $\rho_{\Gamma}$ against $\vartheta=3 u_{0} / R$ are shown for two types of boundary conditions on the cavity surface in Figs. 3-6 $\left(\rho_{0}=r_{0} / R\right)$ for the case when $K_{+}=K_{-}$, $\mu_{+}>\mu_{-}$, that is, when the bulk compression moduli of the material in the two phases are equal and the material which is in the minus phase state has the smaller shear modulus.
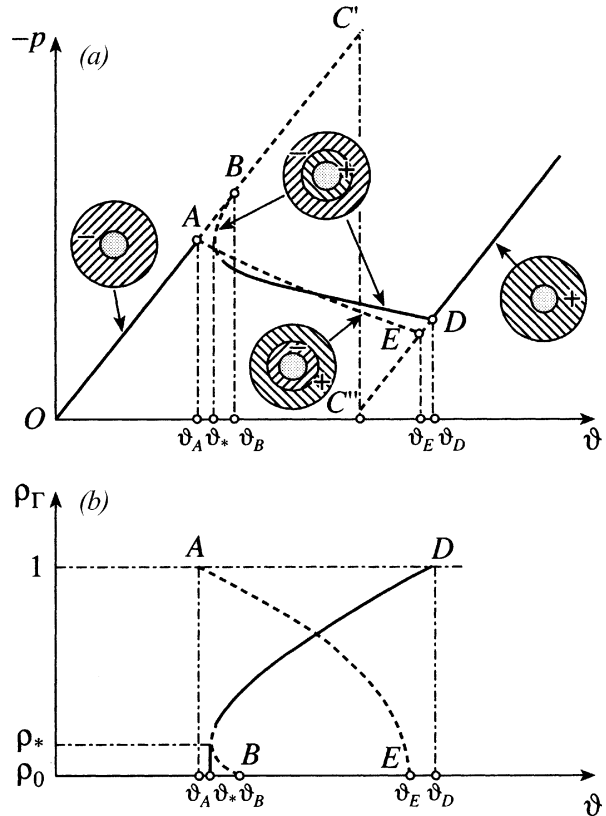

Fig. 4. 

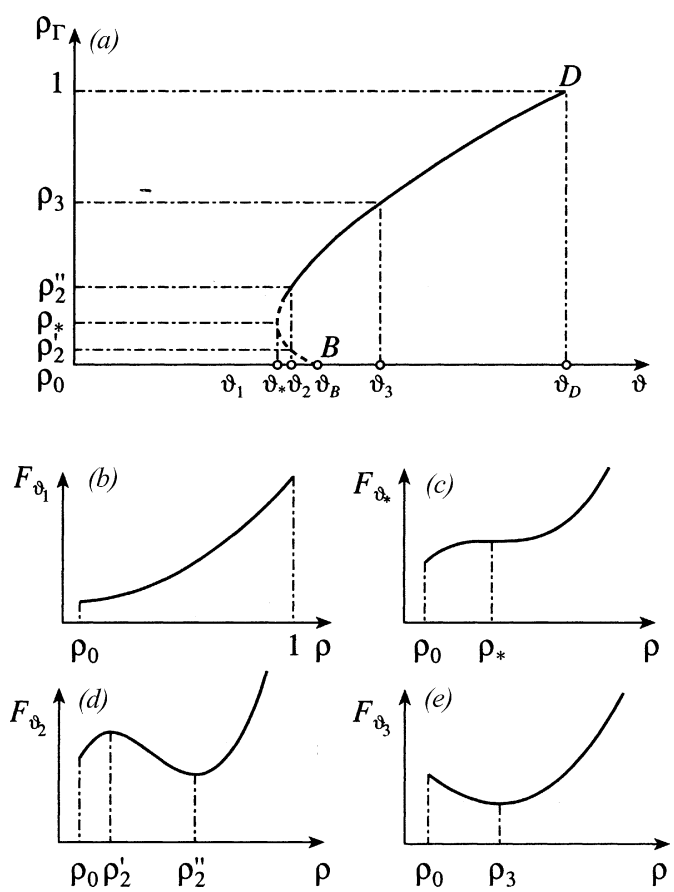

Fig. 5.

\subsection{The surface of a load-free cavity}

Graphs of the pressure $(a)$ and the radii of the equilibrium interfaces $(b)$ as a function of $\vartheta$ are shown in Fig. 3. The curvilinear segments $A E$ and $B D$ correspond to solutions with a single interface. The arrangement of the phase with the greater shear modulus within the interface corresponds to the solution shown by the segment $A E$, and the new phase
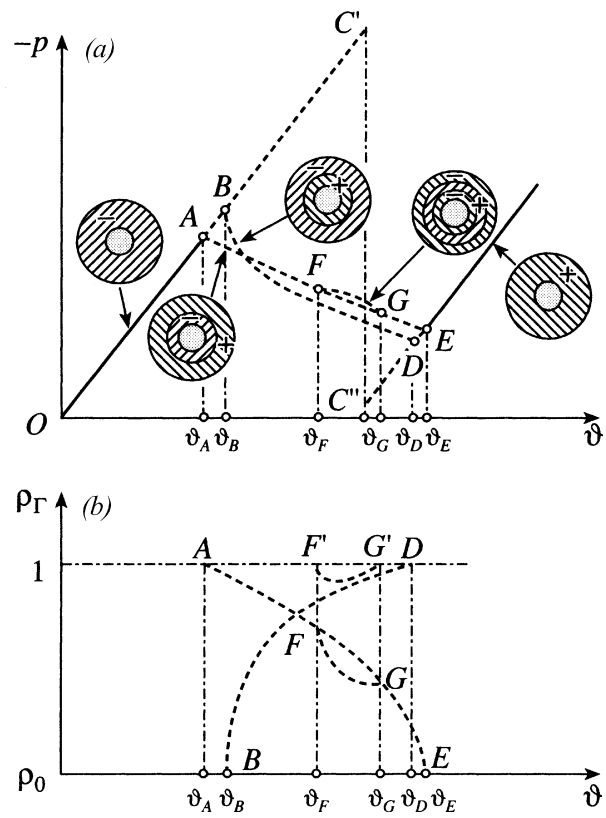

Fig. 6. 
develops from the cavity surface. The development of a more rigid phase from the sphere surface corresponds to the solution shown by the segment $B D$.

When $\vartheta_{F}<\vartheta<\vartheta_{G}$, an equilibrium solution with two interfaces appears, which was missing in the case of a solid sphere. In the graph of the pressure against $\vartheta$, this solution, which is shown by the segment $F G$, branches off when $\vartheta=\vartheta_{F}$ from the second solution with a single interface and terminates at $\vartheta=\vartheta_{G}$ in the line of the first solution with a single interface.

According to this solution, when $\vartheta=\vartheta_{F}$, in a sphere with a cavity there is already either a spherical layer of the more rigid plus phase, bounded by the sphere surface or by the interface of radius $\rho_{F}$ or such a layer is formed (Fig. $3 b$ ). When there is a further increase, the thickness of the layer decreases to zero (the arc $F G$ in Fig. $3 b$ ). A plus phase simultaneously develops from the surface of the cavity (the arc $F G$ ). When $\vartheta=\vartheta_{G}$, a single interface remains in the body and a solution is established which corresponds to the segment $A E$. All three solutions are energy-wise compared preferable with the single phase solution for the same $\vartheta$.

\subsection{Null displacements specified on the surface of the cavity}

A cavity with null displacements in the boundary is equivalent to an absolutely rigid spherical inclusion at the centre of the sphere. For small radii of the inclusion, there are two solutions with a single interface which are analogous to those shown in Fig. 2 for a solid sphere. The energy of the two-phase states, corresponding to the two solutions is less then the energy of the corresponding single-phase state. Both solutions are stable with respect to a perturbation of the radius of the interface.

When the radius of the inclusion is increased, the graphs of the pressure and the radius of the equilibrium interface take the form shown in Fig. 4. The deformation diagram, and the graph of the radius of the interface against $\vartheta$ for the solution with the greater shear modulus of the internal phase, change (the curvilinear segments $B D$ in Fig. $4 a$ and $b$ respectively). A segment of indeterminacy, associated with the radius $\rho^{*}$ of the critical formation of a new phase appears on both curves. This feature was not observed in the case of a load-free cavity. The second solution with a single interface is denoted by means of the dashed segments $A E$. It is also a solution with two interfaces which is unfavourable energy-wise compared with the single-phase states and is therefore not shown.

The results of an investigation into the indeterminacy of the solution corresponding to the segment $B D$ in Fig. 4 are shown in Fig. 5 from the point of view of the minimization of the energy of a two-phase sphere with respect to the radius of the interface. The energy of a two-phase sphere with an arbitrary radius of the interface $\rho$ (with no regard to the thermodynamic condition) is determined by the formula

$$
F=4 \pi R^{3}\left(\int_{\rho_{0}}^{\rho} f_{+}(\varepsilon) \rho^{\prime 2} d \rho^{\prime}+\int_{\rho}^{1} f_{-}(\varepsilon) \rho^{\prime 2} d \rho^{\prime}\right)
$$

and, for a specified value of $\vartheta$ and conditions on the boundary of the cavity, depends on the radius of the interface $\rho$. The equilibrium radius of the interface $\rho_{\Gamma}$ corresponds to the extremum in this relation. The dependence of the radius of the equilibrium interface on $\vartheta$ is shown in Fig. $5 a$ and the characteristic values of $\vartheta$ are labelled. Graphs of the energy of a two-phase sphere $F_{\vartheta}$ against $\rho$ for these characteristic values are shown in Fig. $5 b-e$.

If $\vartheta=\vartheta_{1}$, the graph does not have an extremum when $\rho_{0}<\rho<1$ and, consequently, there is no equilibrium twophase state (Fig. 5b). When $\vartheta=\vartheta *$, a point of inflection $\rho=\rho_{*}=\rho_{\Gamma}$ appears at which $\partial F(\rho, \vartheta) / \partial \rho=0$ (Fig. $5 c$ ). The corresponding energy of the single-phase state is less than the energy of the two-phase state: $F_{\vartheta_{*}}\left(\rho_{0}\right)<F_{\vartheta_{*}}\left(\rho_{*}\right)$. When $\vartheta=\vartheta_{2}$, there are two extrema corresponding to the two equilibrium interfaces $\rho_{2}^{\prime}$ and $\rho_{2}^{\prime \prime}$. The radius $\rho_{2}^{\prime}$ yields a local energy maximum and, consequently, corresponds to a solution which is unstable with respect to perturbations of the radius of the interface. The radius $\rho_{2}^{\prime \prime}$ ensures a minimum of the energy (Fig. $5 d$ ). When $\vartheta=\vartheta_{B}$, the graph of the energy against $\rho$ only has a single extremum, a minimum, corresponding to the equilibrium interface (Fig. 5e).

Hence, when $\vartheta *<\vartheta<\vartheta_{B}$, there are two solutions, but the solution denoted in Fig. $5 a$ by the dashed line is unstable. It is obvious that a value $\vartheta_{M} \in\left(\vartheta_{*}, \vartheta_{2}\right)$ exists for which

$$
F_{\vartheta_{M}}\left(\rho_{0}\right)=F_{\vartheta_{M}}\left(\rho_{M}\right), \quad \partial F_{\vartheta_{M}}(\rho) /\left.\partial \rho\right|_{\rho=\rho_{M}}=0
$$


The two-phase solution, corresponding to the segment $B D$, is energy favourable compared with the single-phase solution when $\vartheta_{M}<\vartheta<\vartheta_{D}$. This means that, as $\vartheta$ increases, the formation of a finite spherical layer of the plus phase with a boundary $\rho_{M}$ becomes energy favourable at the instant when the value $\vartheta=\vartheta_{M}$ is reached.

When there is a further increase in the radius of the inclusion, the critical radius $\rho *$ becomes smaller than the radius of the inclusion, and the corresponding solution will be single-valued. Moreover, when the radius of the inclusion is increased, the solution with two interfaces also becomes energey preferable compared with the singlephase states. Graphs of the pressure and the radii of the equilibrium interfaces against $\vartheta$ in the case of a fairly large radius of the inclusion are shown in Fig. 6. Two equilibrium solutions, corresponding to the segments $A E$ and $B D$, are possible with one segment and a single solution, corresponding to the $\operatorname{arcs} F G$ and $F|G|$, with two interfaces. Unlike the case of a cavity which is load-free (Fig. 3), the arc $F G$ in Fig. $6 a$, which corresponds to the solution with two interfaces, starts and terminates on a line which corresponds to the one and the same solution with a single interface. According to this solution, a layer of the initial minus phase is formed on the surface of the two-phase sphere when $\vartheta=\vartheta_{F}$ when $\vartheta$ increases futher, develops and disappears when $\vartheta=\vartheta_{G}$, after which a solution with a single interface, corresponding to the segment $A E$, is established once again. The change in the radius of the interface which arises when $\vartheta=\vartheta_{F}$, is represented in Fig. $6 b$ by the arc $F|G|$. The change in the interface, which already exists when $\vartheta \leq \vartheta_{F}$, is represented by the arc $F G$. It will be shown below that this solution is unstable.

Hence, the non-linearity of boundary-value problem (1.1), (1.2), caused by the presence of an unknown interface, leads to the existence of several equilibrium solutions even in the class of centrisymmetric deformations. It has been shown earlier for the case of a solid sphere that the energies of the two possible equilibrium two-phase solutions are less than the energies of the single-phase states with the same boundary conditions. ${ }^{18,20}$ The analysis presented above has shown that, in the case of a sphere with a spherical cavity, there is a set of two-phase solutions. the energy of which is less than the energy of the single-phase states and which are stable to perturbations of the radius of the interface.

The problem of choosing of solutions is next considered from the point of view of the metastability of the single-phase states with respect to the formation of domains of a new phase and the local stability of the twophase states with respect to a wider class of perturbations than just perturbations of the radius of the spherical interface.

\section{The formation of a new phase}

The single-phase solutions, represented in Figs. 2-4 by the segments $A C^{\prime \prime}$ and $C^{\prime \prime} E$, are stable in the small in the class of single-phase deformations. The points $A, B, D$ and $E$ (Figs. 2-4) and the points $F$ and $G$ (Fig. 3b) correspond to qualitative changes in the solution and, in fact, when $\vartheta_{A}, \vartheta_{E}, \vartheta_{B}, \vartheta_{D}, \vartheta_{F}, \vartheta_{G}$ are reached, a change occurs in the topology of the solution, that is, the appearance of an equilibrium internal interface becomes possible.

We will investigate the nature of the energy changes accompanying the formation of domains of a new phase before and after these characteristic points have been reached. For one and the same values of $\vartheta$, we compare the energies $F_{-}$ and $F_{+}$of a single-phase sphere in the minus and plus phase states with the energy $F$ of a sphere consisting of a nucleus of the plus phase and a spherical layer formed by the minus phase for arbitrary radii of the nucleus $\rho$, including when the radius is equal to the radius of the equilibrium interface.

The difference in the potential energies of the two-phase and single-phase bodies for the one and the same boundary conditions in the case of an arbitrary (regardless of the thermodynamic condition) interface can be represented in the form 20

$$
F-F_{-}=\int_{V_{+}}\left(\gamma_{*}+\frac{1}{2} \mathbf{q}_{+}: \mathbf{C}_{1}^{-1}: \mathbf{q}_{0}\right) d V ; \quad \mathbf{q}_{0}=-\mathbf{C}_{1}: \boldsymbol{\varepsilon}_{0}+\left[\mathbf{C}: \boldsymbol{\varepsilon}^{p}\right]
$$

where $\varepsilon_{0}$ is the deformation which there would be at the corresponding point of a single-phase body in the case of the same boundary conditions, and the tensor $\mathbf{q}_{+}$is determined by the deformations in the domain $V_{+}$of an arbitrary two-phase body according to formula (1.8). 
In the case of a two-phase sphere, when the plus phase forms a spherical inclusion of arbitrary radius $\rho$ at the centre then, according to (3.6), $\mathbf{q}_{+}=q \mathbf{E}$, where

$$
q=K_{+} \vartheta^{p}-3 K_{1} A_{1}, A_{1}=\frac{d_{-} \vartheta+K_{+} \vartheta^{p}\left(1-\rho^{3}\right)}{3\left(d_{+}-K_{1} \rho^{3}\right)}
$$

and $d_{ \pm}$are defined by formulae (3.11). Substituting the expressions for $\mathbf{q}_{+}$and $\mathbf{q}_{0}$ into equality (5.1), we obtain

$$
F-F_{-}=\frac{4}{3} \pi \rho^{3}\left(\gamma_{*}+\frac{1}{2} q(\tilde{\vartheta}-\vartheta)\right), \quad \tilde{\vartheta}=\frac{K_{+}}{K_{1}} \vartheta^{p}
$$

and, by formulae (5.2), $q$ depends on $\rho$.

Graphs of the difference in the energies as a function of $\rho$ for different $\vartheta$ in the case when $\mu_{1}>0$ (the plus phase has the greater shear modulus) are given in Fig. $7 a$. When $\vartheta>\vartheta_{A}$, the energy of a homogeneous sphere in the minus phase state is less than the energy of a composite sphere for all $\rho \in[0,1]$. There is a minimum in the graph of the difference in the energies of the two-phase and single-phase spheres, but when $\rho<0$.

Suppose $\vartheta_{C}$ corresponds to the intersection of the parabolae $f_{+}(\vartheta)$ and $f_{-}(\vartheta)$, that is, to a change in the domains of definition of the phases (see (1.3) and (1.6)) and $\rho_{\Gamma}^{c}<1$ is the radius of the equilibrium interface when $\vartheta=\vartheta_{C}$. Then, $\vartheta_{A}<\vartheta<\vartheta_{C}$ for all $\rho \in\left[0, \rho_{\Gamma}^{c}\right]$ and the energy of the two-phase sphere is less than the energy of the single-phase sphere in the minus phase state and, at the same time, a minimum, corresponding to the radius $\rho=\rho_{\Gamma}(\vartheta)$ of the equilibrium interface, appears on the graph of the difference in the energies as a function of the radius of the nucleus. When $\vartheta=\vartheta_{A}$, a minimum is reached when $\rho=\rho_{\Gamma}=0$ and, at the same time, $F=F_{-}$.

Similar results can be obtained when comparing the energies of a homogeneous sphere in the plus phase state and a two-phase sphere in the neighbourhood of the point $\vartheta_{E}$ (Fig. 7b) When $\vartheta_{C}<\vartheta<\vartheta_{E}$, the dependence of $F=F_{+}$on $\rho$ has a minimum, corresponding to the equilibrium interface. When $\vartheta=\vartheta_{E}$, a minimum is reached when $\rho=\rho_{\Gamma}=1$ and, at the same time, $F=F_{+}$. If $\vartheta>\vartheta_{F}$, then $F>F_{+}$for all $\rho \leq 1$.
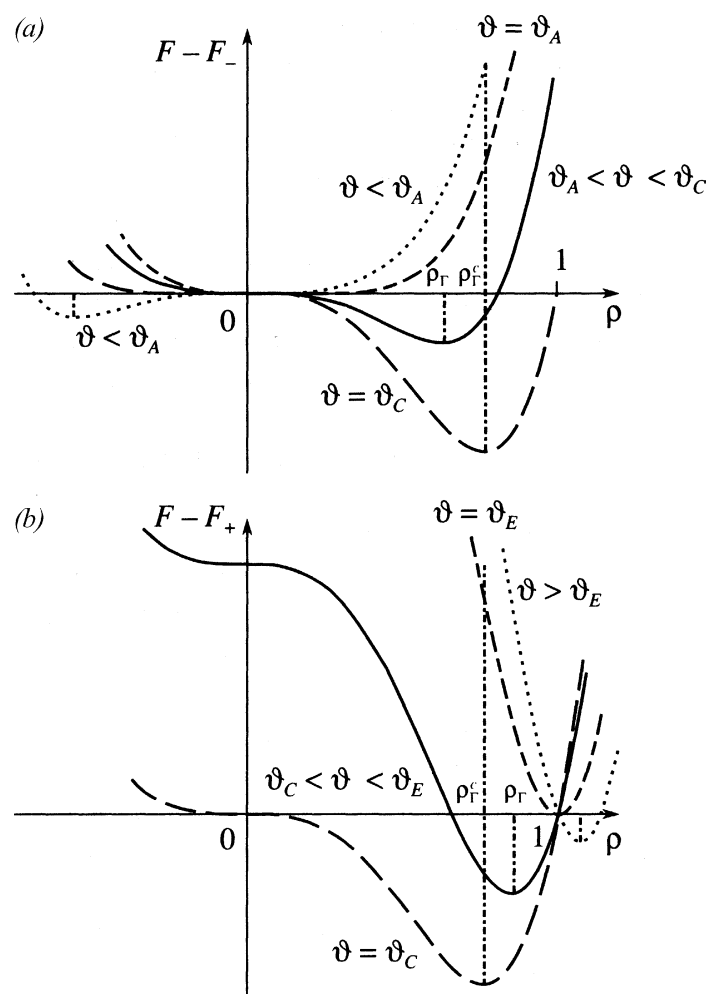

Fig. 7. 
Hence, equilibrium two-phase states are only energy favourable when $\vartheta \in\left[\vartheta_{A}, \vartheta_{E}\right]$. In this range of $\vartheta$, the singlephase solutions are metastable with respect to the two-phase solutions, that is, they are unstable to finite two-phase perturbations. In fact, centrisymmetric two-phase states appear in the neighbourhood of the points $\vartheta_{A}$ and $\vartheta_{E}$ which are more favourable energywise than the single-phase states, even regardless of the thermodynamic condition. The points $\vartheta_{A}$ and $\vartheta_{E}$ can be called points of topological instability (bifurcations).

\section{The local stability of centrisymmetric deformations}

Following the approach described earlier, ${ }^{37}$ we will investigate the stability of the centrisymmetric solutions (CSS) which have been found with respect to axisymmetric perturbations, assuming that

$$
\mathbf{u}=\mathbf{u}^{0}(r)+\mathbf{w}(r, \theta), \quad r=r^{0}+\eta(\theta), \mathbf{w}(r, \theta)=u(r, \theta) \mathbf{e}_{r}+v(r, \theta) \mathbf{e}_{\theta}
$$

Here, $\mathbf{u}^{0}(r)=\mathbf{u}^{0}(r) \mathbf{e}$ is the equilibrium solution of system (3.7), $\mathbf{e}_{r}, \mathbf{e}_{\theta}$ are the basis unit vectors corresponding to the spherical coordinates $r, \theta, \mathbf{w}$, and $\eta$ are the perturbations of the strain field and of the interface respectively. We now write down the linearized equilibrium equations (2.1) for the perturbations

$$
\begin{aligned}
& (\lambda+2 \mu) \frac{\partial \phi}{\partial r}-\frac{2 \mu}{r \sin \theta} \frac{\partial(\omega \sin \theta)}{\partial \theta}=0, \quad(\lambda+2 \mu) \frac{\partial \phi}{\partial \theta}+2 \mu \frac{\partial(r \omega)}{\partial r}=0 \\
& \phi \triangleq \nabla \cdot \mathbf{w}=\frac{\partial u}{\partial r}+2 \frac{u}{r}+\frac{1}{r \sin \theta} \frac{\partial(v \sin \theta)}{\partial \theta}, \quad \omega=\frac{\partial v}{\partial r}-\frac{1}{r}\left(\frac{\partial u}{\partial \theta}-v\right)
\end{aligned}
$$

The force boundary conditions on the surface of the cavity in the case of dead loading (loads which do not change during deformation) take the form

$$
\lambda \phi+2 \mu \frac{\partial u}{\partial r}=0, \quad \omega+\frac{1}{r}\left(\frac{\partial u}{\partial \theta}-v\right)=0
$$

In the case of kinematic boundary conditions on the cavity surface, the vector of the small additional displacements

$$
u=0, \quad v=0
$$

vanishes.

The kinematic and static conditions at the interface, when account is taken of its perturbation, are given by the formulae

$$
\begin{aligned}
& {[u]=-\eta\left[\frac{d u^{0}}{d r}\right], \quad[v]=0} \\
& {\left[\lambda \phi+2 \mu \frac{\partial u}{\partial r}\right]=-\eta\left[\frac{d \sigma_{r}^{0}}{d r}\right], \quad\left[2 \mu\left\{\omega+\frac{1}{r}\left(\frac{\partial u}{\partial \theta}-v\right)\right\}\right]=\frac{d \eta}{d \theta}\left[\sigma_{\theta}^{0}\right]}
\end{aligned}
$$

When $\eta=0$, the last relations are matching conditions at the boundary of separation of the parts of a composite body.

Following the well-known approach in ${ }^{49}$ we will seek a solution of Eq. (6.1) in the form of expansions in Legendre polynomials $P_{n}$ (summation from $n=2$ to $\eta=\infty$ ).

$$
u(r, \theta)=\sum U_{n}(r) P_{n}(\cos \theta), v(r, \theta)=\sum V_{n}(r) P_{n}^{\prime}(\cos \theta) \sin \theta, \eta(\theta)=\sum \zeta_{n} P_{n}(\cos \theta)
$$

Substituting of the expansions into system (6.1) we can separate the variables $r$ and $\theta$.

Using the equalities

$$
\begin{aligned}
& \phi=\sum \Phi_{n} P_{n}(\cos \theta), \quad 2 \omega=\sum \Omega_{n} P_{n}^{\prime}(\cos \theta) \sin \theta \\
& \Phi_{n}=U_{n}^{\prime}+2 \frac{U_{n}}{r}+n(n+1) \frac{V_{n}}{r}, \quad \Omega_{n}=V_{n}^{\prime}+\frac{U_{n}+V_{n}}{r}
\end{aligned}
$$


the equilibrium equations (6.1) can be reduced to a system of ordinary differential equations in $\Phi_{n}(r)$ and $\Omega_{n}(r)$

$$
(\lambda+\mu) r \Phi_{n}(r)^{\prime}-\mu n(n+1) \Omega_{n}(r)=0 \quad(\lambda+\mu) \Phi_{n}(r)-\mu\left(r \Omega_{n}(r)\right)^{\prime}=0
$$

the solution of which is easily found:

$$
\Phi_{n}=(n+1) A_{n} r^{n}-\frac{n B_{n}}{r^{n+1}}, \quad \Omega_{n}=\frac{\lambda+2 \mu}{\mu}\left(A_{n} r^{n}+\frac{B_{n}}{r^{n+1}}\right)
$$

where $A_{n}$ and $B_{n}$ are arbitrary constants.

Solving system (6.5) for $U_{n}$ and $V_{n}$, we obtain the general solution

$$
U_{n}=-n \mathscr{C}_{n}(r) r^{(n-1)}+(n+1) \frac{\mathscr{D}_{n}(r)}{r^{n+2}}, \quad V_{n}=\mathscr{C}_{n}(r) r^{n-1}+\frac{\mathscr{D}_{n}(r)}{r^{n+2}}
$$

Here,

$$
\begin{aligned}
& \mathscr{C}_{n}(r)=\frac{1}{2 n+1} \int_{a}^{r}\left((n+1) \Omega_{n}(\xi)-\Phi_{n}(\xi)\right) \frac{d \xi}{\xi^{n-1}}+C_{n} \\
& \mathscr{D}_{n}(r)=\frac{1}{2 n+1} \int_{a}^{r}\left(\Phi_{n}(\xi)+n \Omega_{n}(\xi)\right) \xi^{n+2} d \xi+D_{n}
\end{aligned}
$$

where $a$ is an arbitrary value of $r$, and $C_{n}$ and $D_{n}$ are constants (which depend, generally speaking, on the choice of $a$ ).

Hence, the general solution of the equilibrium equations in each of the phases is determined by the set of four constants of integration $A_{n}, B_{n}, C_{n}, D_{n}$, which are to be determined from the corresponding boundary conditions.

The variables $r$ and $\theta$ also separate under the conditions on the interface. The linearized conditions (6.2) take the form

$$
\begin{aligned}
& {\left[U_{n}\right]=-\zeta_{n}\left[\frac{d u^{0}}{d r}\right], \quad\left[V_{n}\right]=0} \\
& {\left[\lambda \Phi_{n}+2 \mu U_{n}^{\prime}\right]=-\zeta_{n}\left[\frac{d \sigma_{r}^{0}}{d r}\right], \quad\left[\mu\left(\Omega_{n}-2 \frac{U_{n}+V_{n}}{r}\right)\right]=\frac{\zeta_{n}}{r}\left[\sigma_{\theta}^{0}\right]}
\end{aligned}
$$

Conditions (6.8) with the boundary conditions on the surface of the sphere and on the surface of the cavity (or the condition that the displacements at the centre are bounded in the case of a solid sphere) enable us to find $A_{n}, B_{n}, C_{n}$, $D_{n}$ analytically as functions of the amplitude of the perturbations of the interface $\zeta_{n}$ and the parameters of the initial state. The solution found will be unique by virtue of the uniqueness of the solutions of boundary-value problems in the linear theory of elasticity. The expressions for $A_{n}, B_{n}, C_{n}, D_{n}$ are not presented here because of their length.

\subsection{The stability of two-phase deformations of a solid sphere}

In the case of a solid sphere, the kinematic equation (2.6) takes the form

$$
\begin{aligned}
& q_{ \pm}\left(-\phi\left(1+\frac{\lambda_{1}}{\mu_{\mp}}\left(a_{\mp}-1\right)\right) \mp \frac{2 \mu_{1}}{\mu_{\mp}}\left(a_{\mp}-1\right) \frac{\partial u}{\partial r}\right)=-\frac{1}{k} \frac{d \eta}{d t} \\
& q_{ \pm}=-3 K_{1} A^{ \pm}+K_{+} \vartheta^{p}, \quad a_{ \pm}=\frac{1}{2\left(1-v_{ \pm}\right)}
\end{aligned}
$$

and, after substituting of the expansions (6.3) and (6.4) and separating out the angular coordinate, it can be rewritten as

$$
l_{1} \Phi_{n}\left(r_{0}\right)+l_{2} U_{n}^{\prime}\left(r_{0}\right)=-\frac{1}{k} \frac{d \zeta_{n}}{d t}
$$

where $l_{1}$ and $l_{2}$ are coefficients which depend on the initial state and the moduli of elasticity of the phases. 
After substituting of the coefficients $A_{n}, B_{n}, C_{n}, D_{n}$ as functions of the amplitude of the perturbations of the interface $\zeta_{n}$ and the parameters of the initial state, the kinetic equation becomes

$$
\frac{1}{k} \frac{d \zeta_{n}}{d t}=L_{n}(\vartheta) \zeta_{n}, \quad n=2,3, \ldots
$$

The analytical expression for $L_{n}(\zeta)$ is extremely lengthy and is not presented here. The roots $\vartheta^{*}$ of the equation $L_{n}(\zeta)=0$ are the bifurcation points of boundary-value problem (2.1)-(2.5). The behaviour of small perturbations in the neighbourhood of a bifurcation point depends on the sign of the coefficient $L_{n}$ in Eq. (6.9). Negative values of $L_{n}$ correspond to an amplitude of the perturbations of the interface which is decaying exponentially with time. Positive values of $L_{n}$ correspond to the growth of the perturbations. The number $n$ characterizes the form of loss of stability.

We will call the phase with the greater shear modulus the "hard" phase and that with the smaller shear modulus the "soft" phase. An initial single-phase minus state corresponds to a hard phase $\left(\mu_{-}>\mu_{+}\right)$or a soft phase $\left(\mu_{-}<\mu_{+}\right)$.

The graphs of $L_{n}$ (the solid lines) and $\rho_{\Gamma}$ (the dashed lines) against $\vartheta / \vartheta^{\rho}$ are shown in Fig. 8 for the case when $\mu_{+}>\mu_{-}$. Figure $8 a$ corresponds to the two-phase equilibrium solution in which the new phase develops from the centre of the sphere. This solution is represented in Fig. 2 by the segment $A E$. Figure $8 b$ corresponds to the second solution (the segment $B D$ in Fig. 2), according to which the hard phase develops from the surface of the sphere. In the first case, no instability was revealed. In the second case, there is a loss of stability, in the first place by a high frequency mode, practically immediately upon the emergence of a thin spherical layer formed by the plus phase.

The results of the analysis of the stability of a two-phase sphere for $\mu_{+}<\mu_{-}$, when the new soft phase is generated at the centre of the sphere, are shown in Fig. 9. As the domain of the new phase develops, there is a loss of stability through a low-frequency mode. No instability was revealed in the case when the new phase develops from the surface of the sphere.

As a whole, the results presented in Figs. 8 and 9 enable us to draw a conclusion concerning the local instability of a two-phase deformation in the case when the soft phase is located within the sphere.

Hence, the appearance of a new degree of freedom associated with the existence of an interface which was unknown in advance has a considerable effect on the stability of two-phase bodies. When there is no phase transition (when $\eta \equiv 0$ ), the boundary-value problem (2.1)-(2.5) is a well known homogeneous boundary-value problem for a composite linear elastic soild (a composite) which only has null solutions. This means that a centrisymmetric solution of a problem without a phase transition is always stable.
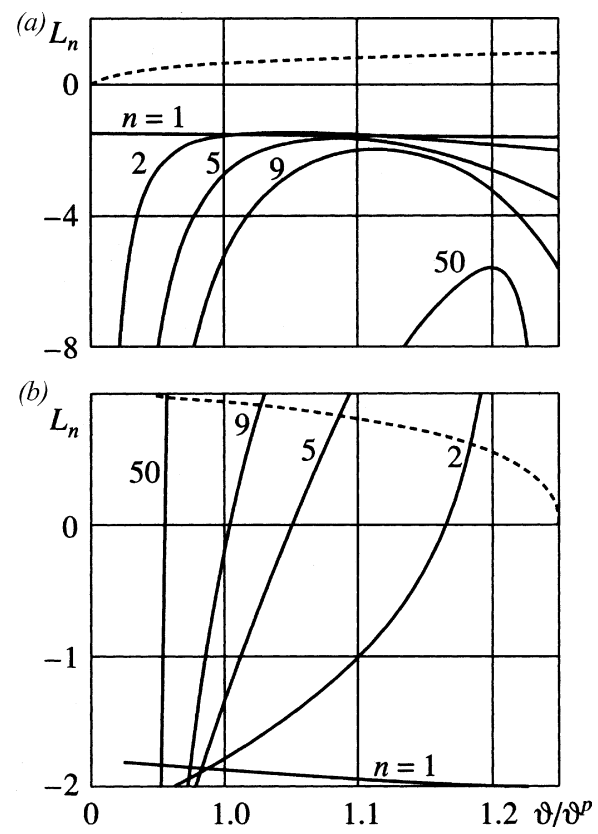

Fig. 8 . 


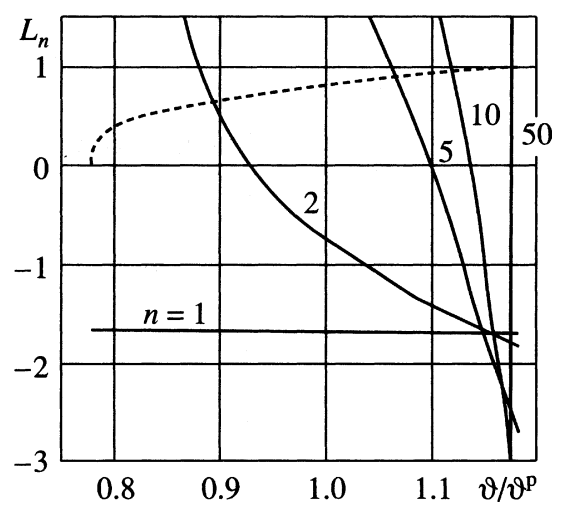

Fig. 9.

\subsection{The stability of a two-phase sphere with a cavity or a hard inclusion at the centre}

It has been shown above that, in the case of a sphere with a cavity which undergoes a phase transformation, no more than two equilibrium spherical interfaces can exist. The investigation of the loss of stability of centrisymmetric deformations does not differ fundamentally from the case of a solid sphere with a single interface. The additional technical complexities are associated with the increase in the number of unknowns and, correspondingly, in the number of equations.

We next assume that the bulk compression moduli of the material are the same in both phases: $K_{-}=K_{+}=K$. In this case, kinetic equation (2.5) takes the form

$$
\begin{aligned}
& \phi\left(-q_{1} \pm \frac{2 \mu_{1}}{3 d_{\mp}^{0}}\left(q_{1}+q_{2}\right)\right)-\frac{\partial u}{\partial r}\left(q_{2} \pm \frac{2 \mu_{1}}{d_{\mp}^{0}}\left(q_{1}+q_{2}\right)\right)=-\frac{1}{k} \frac{d \eta}{d t} \\
& q_{1}=-\frac{2 \mu_{1} D_{ \pm}}{r^{3}}+K \vartheta^{p}, \quad q_{2}=\frac{6 \mu_{1} D_{ \pm}}{r^{3}}, \quad d_{ \pm}^{0}=K+\frac{4}{3} \mu_{ \pm}
\end{aligned}
$$

where the upper (lower) plus or minus sign corresponds to the option of deformations at the interface from the direction of the plus phase (the minus phase). After substituting expansions (6.3) and (6.4) and separating out the angular coordinate, this equation reduces to the form

$$
\Phi_{n}^{ \pm}\left(-q_{1} \pm \frac{2 \mu_{1}}{3 d_{\mp}^{0}}\left(q_{1}+q_{2}\right)\right)-U_{n}^{ \pm}\left(q_{2} \pm \frac{2 \mu_{1}}{d_{\mp}^{0}}\left(q_{1}+q_{2}\right)\right)=-\frac{1}{k} \frac{d \zeta_{n}}{d t}
$$

The case of a single interface is investigated in a similar manner to that for the case of the interface in a solid sphere. In the case of two interfaces, the solution of the equilibrium equation for a three-layer sphere is given by formulae of the type of (6.6) and (6.7) and is determined by a set of 12 constants of integration three quartets of constants $A_{n}$, $B_{n}, C_{n}, D_{n}$ for the external, intermediate and internal phases. Boundary conditions of the form of (6.8) serve for their determination. The stability analysis reduces to an investigation of the behaviour of the amplitudes $\zeta_{n}^{(1)}$ and $\zeta_{n}^{(2)}$ of the perturbation of the interface with time, which is described by a system of ordinary differential equations obtained from kinetic equations (6.10)

$$
\frac{d \zeta_{n}^{(1)}}{d t}=L_{n}^{(11)}(\vartheta) \zeta_{n}^{(1)}+L_{n}^{(12)}(\vartheta) \zeta_{n}^{(2)}, \quad \frac{d \zeta_{n}^{(2)}}{d t}=L_{n}^{(21)}(\vartheta) \zeta_{n}^{(1)}+L_{n}^{(22)}(\vartheta) \zeta_{n}^{(2)} ; \quad n=2,3, \ldots
$$

For the stability of a centrisymmetric deformation with two interfaces, it is necessary that the matrix of the coefficients $L_{n}^{i k} \quad(i, k=1,2)$ should be negative definite. The values of the parameter $\vartheta$ at which the matrix degenerates, are the bifurcation points.

The analysis carried out shows that, in the case of a cavity of small dimensions which is unloaded, a solution with a single interface can be stable provided that the phase with the greater shear modulus forms a spherical layer 
adjacent to the cavity (the solution corresponding to $A D$ in Fig. 3). The remaining solutions are unstable. When the radius of the cavity increases $\left(\rho_{0}>10^{-2}\right.$ for the material parameters chosen), all the two-phase solutions become unstable.

We will now consider the stability of two-phase solutions for a sphere with an absolutely rigid inclusion at the centre. The equilibrium conditions, generally speaking, permit three solutions with a different alternation of the phases. We next assume that $\mu_{+}>\mu_{-}$.

If the radius of the inclusion is very small $\rho_{0}<10^{-2}$, then loss of stability is not revealed from two of the available solutions in the case of the solution for which the plus phase develops from the surface of the sphere. The second solution with a single interface is unstable.

When the radius of the inclusion is increased, a hard plus phase is formed on the surface of the inclusion in the form of a spherical layer of critical radius $\rho_{\mathbf{M}}$ or develops from the surface of the sphere. The second solution is unstable. We recall that, in this case, the nucleation of the plus phase originates when $\vartheta=\vartheta_{\mathbf{M}} \in\left(\vartheta_{*}, \vartheta_{2}\right)$ (Fig. 4).

When the radius of the inclusion increases further $\left(\rho_{0} \sim 10^{-1}\right)$, the first two-phase solution also loses stability through a low-frequency mode, and the number of modes through which the first loss of stability occurs increases as the radius of the inclusion increases. This tendency is also maintained when the radius of the inclusion increases further when the critical radius of the nucleation of the new phase becomes less than the radius of the inclusion.

At large radii of the inclusion, a solution is also possible with two interfaces with alternation of the minus - plus minus phases. However, this solution is unstable with respect to both perturbations of the radius of the interface and, also, to axisymmetric perturbations.

Hence, the appearance of a cavity turns out to have a destabilizing effect regardless of the type of boundary conditions which are specified for it. For small cavities, a two-phase solution with a single interface, which describes the case of the arrangement of the harder phase around the cavity, turns out to be stable. By comparing the results of the investigation of the stability for a sphere with a cavity and a solid sphere, it can be seen that the stable solution when the radius of the cavity tends to zero passes into the stable solution for the solid sphere.

In the case of a cavity of larger dimensions with a fixed surface, all the two-phase centrisymmetric solutions turn out to be unstable in the small. This implies the need to look for more complex solutions with non-spherical interfaces.

Energy favourable two-phase centrisymmetric solutions which are stable in the small are shown by the solid line in Figs. 2-4. Unstable two-phase solutions and metastable solutions are denoted by the dashed lines.

Considerations of energy efficiency and stability in the small therefore enable us to select a unique solution in the class of centrisymmetric solutions.

\subsection{Comparison of the solutions of the problem of a two-phase composite with phase transitions}

The solutions of the problem of equilibrium two-phase configurations of elastic bodies can differ from the solutions obtained in the case of two-phase composite materials or composite bodies of optimal structure. Centrisymmetric solutions have been considered ${ }^{50}$ which convey a global minimum to the energy functional of a two-phase body in the case of a fixed fraction of one of the phases. The phases differed solely in their moduli of elasticity which corresponds to the case when $\varepsilon^{p}=0$. It has been shown, in the case of a continuous sphere, that the structure, in which the hard phase is concentrated at the centre of the sphere and is surrounded by a soft phase, corresponds to the solution that furnishes the global minimum, which is identical to the results of the stability investigation presented above. However, in the case of a sphere with a cavity with different moduli of bulk compression of the material of the phases, the solution which is a three-layer shell, the middle layer of which is formed by the hard phase, is optimal, that is, energy favourable in the class of states which satisfy the isoperimetric condition of constancy of the composition. As has been shown above, in the case of phase transitions, this solution turns out to be unstable on account of the additional degree of freedom associated with the possibility of a change in the relative content of the phases as a consequence of the transition.

\section{Equilibrium two-phase deformations and zones of phase transitions}

We will now consider how the strain fields in the different two-phase configurations of a sphere with a zone of phase transitions (ZPT), formed by all the deformations which can exist at the equilibrium interface, are related. ${ }^{16,24}$ 
The boundaries of the ZPT are determined by the material parameters. In the case of small deformations, the ZPTs are constructed on the basis of a thermodynamic equilibrium condition in the form of (1.9) or, if the tensor $\mathbf{C}_{1}$ is nondegenerate, in the form of (1.10). In the latter case, the ZPT can be constructed in $q$-space, that is, in the space of the tensors $q=-\mathbf{C}_{1}: \boldsymbol{\varepsilon}+\left[\mathbf{C}: \varepsilon^{p}\right]$. It follows from condition (1.10) that the tensors $\mathbf{q}_{ \pm}$, which can correspond to deformations at the equilibrium interface, must satisfy inequalities which determine the subzones of the plus and minus phase transitions in $q$-space ${ }^{19,21}$ :

$$
\begin{aligned}
& K_{\min }^{\mp}\left(\mathbf{q}_{ \pm}\right) \leq \pm \varphi\left(\mathbf{q}_{ \pm}\right) \leq K_{\max }^{\mp}\left(\mathbf{q}_{ \pm}\right) \\
& \varphi\left(\mathbf{q}_{ \pm}\right)=2 \gamma_{*}+\mathbf{q}_{ \pm}: \mathbf{C}_{1}^{-1}: \mathbf{q}_{ \pm}, \quad K_{ \pm}(\mathbf{q}, \mathbf{n})=\mathbf{q}: \mathbf{K}_{ \pm}(\mathbf{n}): \mathbf{q} \\
& K_{\max }^{\mp}\left(\mathbf{q}_{ \pm}\right)=\max _{\mathbf{n}} K_{\mp}(\mathbf{q}, \mathbf{n}), \quad K_{\min }^{\mp}\left(\mathbf{q}_{ \pm}\right)=\min _{\mathbf{n}} K_{\mp}\left(\mathbf{q}_{\mp}, \mathbf{n}\right)
\end{aligned}
$$

The construction of ZPTs has been considered earlier in Refs. 19,21,26 and 27 for the case of isotropic phases. If the tensor $\mathbf{C}_{1}$ is of fixed sign, the ZPTs are bounded by closed surfaces. If the tensor $\mathbf{C}_{1}$ is not of fixed sign, the ZPTs are open. If the deformation at the interface corresponds to the boundary of a ZPT, then the normal to the interface and the discontinuity in the deformations are uniquely defined by the deformation at one of the sides of the interface.

In the case of centrisymmetric two-phase deformations of an isotropic material, spherical and axisymmetric fields of the tensor $\mathbf{q}$ arise in the body. In the space of the principal values $q_{1}, q_{2}, q_{3}$, these fields lie in the plane $q_{2}=q_{3}$. The intersections of the ZPTs with the plane $q_{2}=q_{3} \equiv \mathrm{q}$ and the strain fields, corresponding to two-phase equilibrium deformations of a solid sphere, are shown in Fig. $10 a\left(\mu_{1}>0, K_{1}<0\right)$ and Fig. $10 b\left(\mu_{1}<0, K_{1}<0\right)$ in the coordinates $z=\operatorname{trq} / \sqrt{3}, q-q_{1}$.

The $z$ axis corresponds to isotropic tension-compression deformations, the point $z_{0}$ corresponds to the undeformed state (the minus phase), the lines $\omega$ are the external boundaries of the ZPTs (the external boundaries of the plus and minus subzones), the lines $\zeta$ are the internal boundaries of the plus and minus subzones, and the dashed line $\xi$ separates the domains of definition of the phases (see relations (1.6)).

Depending on the relation between the principal values of the tensor $\mathbf{q}$, interfaces with a normal which is differently orientated with respect to the principal directions of the tensor $\mathbf{q}$ correspond to the external boundaries of the ZPTs [ Refs. 14,19,21,27]. The parts of the lines $\omega$ which are indicated by a dotted line correspond to interfaces with a normal coinciding with a principal direction which corresponds to the principal value of $\mathbf{q}$ with the greatest modulus. At such interfaces, only the corresponding principal value of the strain tensor is changed by a discontinuity. The parts of $\omega$ denoted by the solid lines correspond to normals which lie in the plane of the greatest and the smallest principal values of $\mathbf{q}$ and, at the corresponding points of the interface, there is a discontinuity in the shear components of the strain tensor; the interface can be identified with the boundary of a shear zone [14,27].

The $z$ axis intersects the ZPT only if $\gamma * K_{1}<0$ (compare with the condition for the existence of centrisymmetric solutions (3.12)). When there are intersections with the $z$ axis, minus and plus subzones degenerate into points. All possible interfaces, on one side of which the tensor $\mathbf{q}$ is spherical, are exhausted by these points. For example, when $z>z_{0}$, only the two interfaces, represented in Fig. 10 by the discontinuities in the deformations $e e^{\prime}$ and $b b^{\prime}$, are permitted. These interfaces correspond to the two solutions of the problem of a solid sphere when the internal spherical domain is in a plus phase state (point $e$ ) or minus phase state (point $b$ ) considered above. The existence and position of the points $e, e^{\prime}, b$ and $b^{\prime}$ is exclusively determined by the material parameters.

If $\mu_{1}>0$, in Fig. $10 a$, the point $e$ (the plus phase for a deformation $\vartheta_{E}$ ) and the segment $e^{\prime} a$, which represents the deformation in $q$-space in a spherical layer formed by the minus phase, correspond to the solution $A E$ (Fig. 2) with the greater shear modulus of the internal phase. The segment is perpendicular to the $z$ axis since, in the case of centrisymmetric deformations, $z=$ const within a spherical layer. In $q$-space, motion along the $z$ axis corresponds to a change in the external parameter $u_{0}$. In the deformation path $z_{0} e$, on reaching point $a$, which corresponds to $\vartheta=\vartheta_{A}$ in Fig. $2 a$, the formation of a plus phase at the centre of the sphere becomes possible and an interface with a discontinuity in the deformations $e e^{\prime}$ arises. On further deformation of the sphere, the interface expands. The deformation of the internal plus phase remains unchanged (points $e$ and $E$ in Fig. $10 a$ and Fig. $2 a$ respectively). For each radius of the interface, the deformations in the spherical layer of the minus phase correspond to a segment with a start at the point $e^{\prime}$, which is part of the segment $e^{\prime} a$. The right-hand end of the segment is displaced towards the point $e^{\prime}$ in proportion to the deformation of the sphere and the corresponding change in the radius of the interface. So, in the case of a certain 

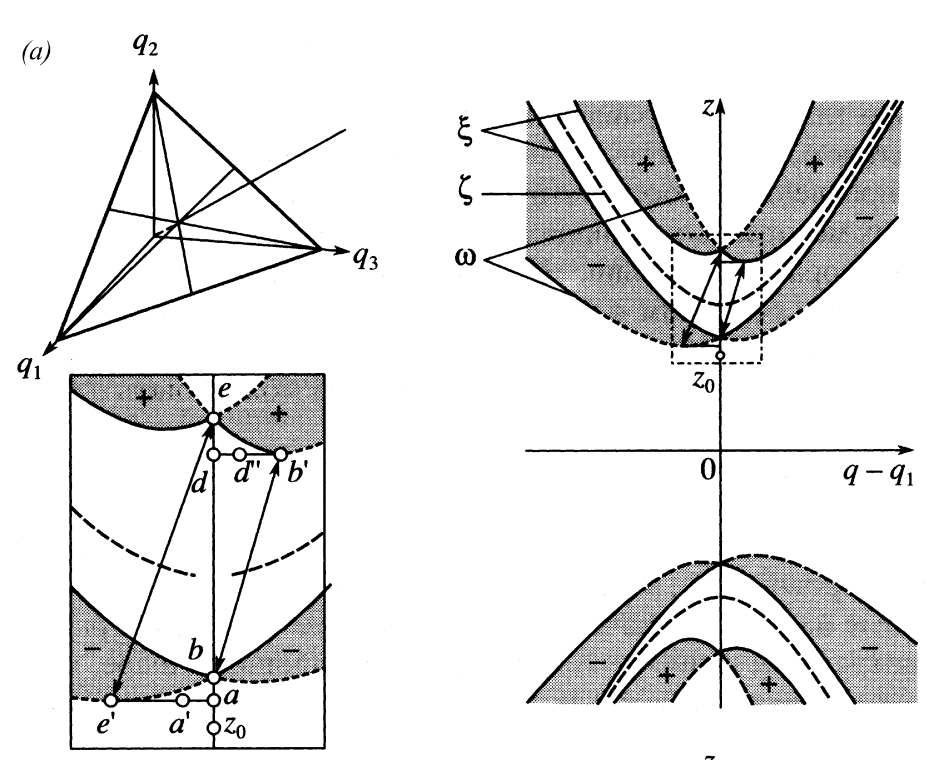

(b)

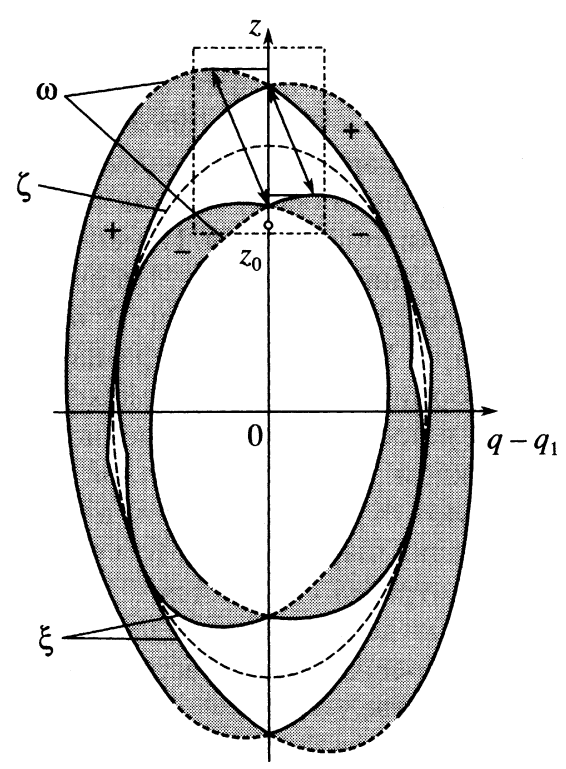

Fig. 10 .

radius of the interface, the deformations in the spherical layer of the minus phase will be distributed along the segment $e a^{\prime}$. The transformation is completed when the mean bulk deformation reaches the point $e$ in $q$-space.

The second solution ( $B D$ in Fig. 2) is represented in Fig. $10 a$ by the point $b$ (the minus phase in the case of a deformation $\vartheta_{B}$ ) and by the segment $b^{\prime} d$, which is perpendicular to the $z$ axis (the plus phase) When the point $b$, corresponding to $\vartheta=\vartheta_{B}$, is reached in the deformation path $z_{0} e$, an infinitesimal small spherical layer of the plus phase arises on the surface of the sphere with a deformation $b^{\prime}$ at the interface which, on further deformation, expands into the sphere. The end $d^{\prime}$ of the segment $b^{\prime} d^{\prime}$, corresponding to deformation of the internal spherical layer, moves from the point $b^{\prime}$ to the $z$ axis as the layer of plus phase develops. The transformation is completed when the mean bulk deformation reaches the point $d$ in $q$-space.

If $\mu_{1}<0$ (Fig. 10b), then an infinitesimal spherical layer of the plus phase is generated when the point $b$ reaches the surface of the sphere. Point $b$ corresponds to the deformation in the internal minus phase. The interface advances into the sphere in proportion to the extent that the sphere is transformed. For each radius of the interface, the deformations in the spherical layer of the plus phase correspond to a segment which is part of the segment $b^{\prime} d$, where the point $b^{\prime}$ corresponds to a deformation at the interface and the right-hand end of the segment moves from point $b^{\prime}$ to the $z$ axis. 
The transformation is complete when the mean bulk deformation corresponds to the point $d$. The second solution is represented in Fig. $10 b$ by the discontinuity $e e^{\prime}$ and the segment $e^{\prime} a$.

Hence, the number of centrisymmetric solutions with a single interface and the corresponding strain fields can be represented if the ZPT is constructed. We shall show that the impossibility of the coexistence in a solid sphere of two or more interfaces is also clear from a direct consideration of the ZPT.

Regardless of the thermodynamic condition in a two-phase sphere with several spherical interfaces, the field trq is piecewise-constant. According to relations (3.5), the quantities $q-q_{1}=6 \mu_{1} D_{i} / r^{3}$ do not change sign within a layer. Consequently, the deformations within the layers correspond in $q$-space to the points of segments which are perpendicular to the $z$ axis and do not intersect this axis.

We assume that a spherical layer exists which is bounded by two equilibrium interfaces with radii $r_{1}<r_{2}$, where the interface $r_{1}$ separates the layer $V_{2}$ from the spherical domain $V_{1}$. The deformations which are permissible at the interface bounding the spherical domain, are prescribed by the properties of the material and are represented by the points $e, e^{\prime}, b, b^{\prime}$, in $q$-space when $z>z_{0}$. Hence, regardless of the existence of other interfaces within a spherical layer, only two values of $z$ are permissible, which are determined by the position of the points $e^{\prime}$ and $b^{\prime}$. The fields within a layer are represented by segments which start out from the points $a^{\prime}$ and $b^{\prime}$, in the direction normal to the $z$ axis and do not intersect the $z$ axis. According to Fig. 10, these segments have no other points in common with the ZPT. Deformations at the equilibrium interface must belong to the ZPT. Consequently, the deformation when $r=r_{2}-0$ must correspond to the points $e^{\prime}$ and $b^{\prime}$, that is, be identical to the deformations when $r=r_{1}+0$. However, then the deformations when $r=r_{2}+0$ must correspond to the point $e$ or $b$ which, in turn, corresponds to a spherical tensor $-q$ like when $r<r_{1}$. The tensor $q$ must therefore be spherical not only in the domain $V_{1}$ but also in the domain $V_{3}$. Substituting $D_{3}=0, A_{3}=A_{1}$ into the first two equations of (3.7), we obtain that $r_{1}=r_{2}$ and $/$ or $D_{2}=0$. The latter means that the tensor $q$ is spherical when $r_{1}<r<r_{2}$ and the same contradiction has been arrived at as in the proof of the theorem.

From a comparison of the strain fields with the ZPTs in the case of a solid sphere with a cavity, it can be concluded that strain fields of two types correspond to the different two-phase states: a) those which lie outside of the external boundaries of the ZPT everywhere, apart from the interface, deformations at which correspond to the external boundary of the ZPT and b) deformations which, at least, in parts of the body, correspond to internal points (boundaries) of the ZPT and there is a discontinuity with a non-convex part of the external boundary of the ZPT.

The analysis of centrisymmetric two-phase deformations has shown that the unstable solutions correspond to the second of the above mentioned types of deformations.

\section{Conclusion}

Our investigation has demonstrated a number of special features of the boundary-value problems of bodies which undergo phase transitions during deformation.

1. The non-linearity of the boundary-value problem caused by an unknown interface leads to non-uniqueness of the solution even in the case of linearly elastic phases.

2. The additional degree of freedom caused by the existence of an interface affects the stability of elastic two-phase bodies. When there is no phase transition, the centrisymmetric deformations of a composite linearly elastic sphere are stable for any radius of the interface.

3. The appearance of a cavity turns out to have a destabilizing action. It was discovered in the case of a cavity with a fixed surface that all the centrisymmetric two-phase solutions can be unstable and that the energy of a two-phase state can be lower than the energy of the corresponding single phase states. This implies the need for a proof of the existence and construction of other two-phase equilibrium solutions.

4. Solutions of the problem of the equilibrium two-phase configurations of elastic bodies can differ from the point of view of the stability of the solutions obtained when designing two-phase composites of optimal structure.

5. The use of zones of phase transitions can be useful for a preliminary estimate of the number of solutions and the characteristic features of two-phase deformations.

\section{Acknowledgements}

We wish to thank N. F. Morozov for frequent discussions of the formulation of the problem and the results obtained. 
This research was supported financially by the Russian Foundation for Basic Research (04/01-00431), the International Association for Promoting Cooperation with Scientist from the New Independent States of the Former Soviet Union (INTAS-03-55-1172). the Program of Basic research of the Division of Energetics, Machine Construction, Mechanics and Control Processes of the Russian Academy of Sciences and the Foundation for Collaboration in National Science and the Federal Agency for Science and Innovation (Mk-826, 2006,1).

\section{References}

1. Likhachev VA, Kuz'min SL, Kamentseva ZP. The Shape Memory Effect. Leningrad: LGU; 1987.

2. Likhachev VA, editor. Shape Memory Materials. St. Petersburg: NIIKh SpbGU; 1998.

3. Boiko VS, Garber RI, Kosevich AM. The Reversible Plasticity of Crystals. Moscow: Nauka; 1991.

4. Gibbs JW. Scientific Papers. V. I. Thermodynamics. N.Y. etc. Dover, 1906 (Gibbs, JW. Termodinamika. Staisticheskaya mekhanika. Moscow:Nauka; 1982.).

5. Grinfel'd MA. The conditions for thermodynamic equilibrium of the phases of a non- linear elastic material. Dokl Akad Nauk SSSR 1973;251(4):824-7.

6. James RD. Finite deformation by mechanical twinning. Arch Rat Mech Anal 1981;77:143-77.

7. Truskinovskii LM. Equilibrium interfaces. Dokl Akad Nauk SSSR 1982;265(2):306-10.

8. Kondaurov VI, Nikitin LV. Phase transitions of the first kind in non-linear elastic media. Dokl Akad Nauk SSSR 1982;262(6):1348-51.

9. Gurtin ME. Two-phase deformations of elastic solids. Arch Rat Mech Anal 1983;84(1):1-29.

10. Kondaurov VI, Nikitin LV. Phase transitions of the first kind in an elastoviscoplastic medium. Izv Akad Nauk SSSR MTT 1986;(4):130-9.

11. Kondaurov VI, Nikitin LV. Thermomechanics of phase transitions in elastoviscoplastic medium at finite deformations. In: Matematical Methods of the Mechanicas of Deformable Solids. Moscow: Nauka; 1986.

12. Fosdick R, Hertog B. The Maxwell relation and Eshelby's conservation law for minimizers in elasticity theory. J Elasticity 1989;22:193-200.

13. Kublanov LB, Freidin AB. Nucleation of a solid phase in a deformable material. Prikl Mat Mekh 1988;52(3):493-501.

14. Freidin AB. Cracks of silver and shear zones in glass-like polymers as layers of a new phase. Mekhanika Kompozitn Materialov 1989;(1):3-10.

15. Grinfel'd MA. Methods of Continuum Mechanics in the Theory of Phase Transitions. Moscow: Nauka; 1990.

16. Freidin AB, Chiskis AM. Zones of phase transitions in non-linear elastic isotropic materials. Part 1. Basic Relations. Izv Akad Nauk MTT 1994;(4):91-109.

17. Freidin AB, Chiskis AM. Zones of phase transitions in non-linear elastic isotropic materials. Part 2. Incompressible materials with a potentials solely dependent on one of the invariants of the strain tensor. Izv Ross Akad Nauk MTT 1994;(5):49-61.

18. Morozov NF, Nazyrov IR, Freidin AB. One-dimensional problem of the phase transitions of an elastic solids. Dokl Ross Akad Nauk 1996;346(2):188-91.

19. Morozov NF, Freidin AB. Zones of phase transitions and phase transformations of elastic solids for different forms of stress state. Tr Mat Inst im V A Steklova 1998;223:220-32.

20. Nazyrov IR, Freidin AB. Phase transitions accompanying the deformation of solids in a model problem of an elastic sphere. Izv Ross Akad Nauk MTT 1998;(5):52-71.

21. Freidin AB. Approximation of small deformations in the theory of phase transitions accompanying the deformation of elastic solids. In: Strength and Fracture of Materials in Constructions (Edited by N. F. Morozov). Research on Elasticity and Plasticity. Issue 18. St. Petersburg: Izd. SPb. Univ.; 1999:266-90.

22. Osmolovskii VG. Variational Problem of Phase Transitions in Continuum Mechanics. St. Petersburg: Izd. Spb. Univ.; 2000.

23. Morozov NF, Freidin AB. Equilibrium two-phase strain fields in elastic solids. In: Proc. 27th Summer School "Analysis and Synthesis of Non-linear Mechanical Oscillatory Systems” St. Petersburg: Repino, 1999. St. Petersburg: IPMash Ross Akad Nauk; 2000:497-516.

24. Freidin AB. The equilibrium of the phases of an isotropic non-linear-elastic material. Izv Vuzov Severo-Kavkaz Region Yestest Nauki Special Issue. Non-linear Problems of Continuum Mechanics 2000:150-68.

25. Vakulenko AA. The micro- and macrokinetics of martensite transitions. Izv Ross Akad Nauk MTT 2001;(5):43-62.

26. Freidin AB, Vilchevskaya EN, Saripove LL. Two-phase deformations within the framework of phase transition zones. Theoret and Apll Mech 2002;28-29:149-72.

27. Freidin AB, Sharipova LL. Equilibrium two-phase deformations and zones of phase transitions in the approximation of small deformations. Izv Vuzov Severo-Kavkaz Region Yestest Nauki. Special Issue Non-linear Problems of Continuum Mechanics; 2003:291-8.

28. Likhachev VA, Malinin VG. Structural Analytical Theory of Stability. St. Petersburg: Nauka; 1993.

29. Movchan AA. A micromechanical approach to the description of the deformation of martensite transitions in shape memory alloys. Izv Ross Akad Nauk MTT 1995;(1):197-205.

30. Movchan AA. Analytical solution of problems on the direct and inverse transitions for shape memory alloys. Izv Ross Akad Nauk MTT 1996;(4):136-44.

31. Yeremeyev VA, Freidin AB, Sharipova LL. Centrisymmetric two-phase strain fields. In Problems of the Mechanics of a Deformable Solids. Interschool Compilation on the 70-th Birthday of N.F. Morozov. St. Petersburg: Izd. SPB. Univ.; 2002:111-12.

32. Yeremeyev VA, Freidin AB, Sharipova LL. Non-uniqueness and stability in problems of the equilibrium of elastic two-phase solids. Dokl Ross Akad Nauk 2003;391(2):189-93.

33. Laudise RA. The Growth of Single Crystals. Eaglewood Cliff: Prentice-Hall; 1970.

34. Lyubov BYa. Theory of Crystallization in Large Volumes. Moscow: Nauka; 1980. 
35. Sekerka RF. Morphological instabilities during phase transformations. Phase Transformations and Material Instabilities in Solids. Madison, Wis., 1983. Publ. Math. Res. Center Univ. Wisconsin, 52. Orlando, FL. Acad. Press, 1984. P. 147-62.

36. Balandin GF. Fundamentals of the Theory of the Formation of Castings. Part 1. Thermal Principles of the Theory. Hardening and Cooling of a Casting. Moscow: Mashinostroyeniye; 1976.

37. Yeremeyev VA, Zubov LM. The stability of the equilibrium of non-linear elastic solids undergoing phase transitions. Izv Akad Nauk SSSR MTT $1991 ;(2): 56-65$.

38. Yeremeyev VA. Bulging of a non-linear elastic plate lying on the liquid surface taking phase transition into account. Zh Prikl Mekh Tekhn Fiz $1991 ;(3): 141-7$.

39. Yeremeyev VA. Equilibrium and stability of micro-inhomogeneous elastic solids undergoing phase transitions. Mat Modelirovanie 1997;9(2):66-9.

40. Sharipova LL, Yeremeyev VA, Freidin AB. The stability of an elastic two-phase sphere. Izv vuzov Svero-Kavkaz Region yestest Nauki. Special Issue. Mathematical Modelling; 2001:166-8.

41. Movchan AA, Sil'chenko LG. The stability of a Shenley column in the case of creep or during a direct thermoelastic transition. Mekh Kompoz. Materialov konstruk 2000;6(1):89-102.

42. Movchan AA, Kazarina SA. Experimental investigations of the phenomenon of loss of stability caused by thermoelastic phase transitions under compressive stresses. Probl Mashinostroyeniya Nadezhnosti Mashin 2002;(6):82-9.

43. Movchan AA, Sil'chenko LG. The stability of a rod undergoing a direct or reverse martensite transition under compressive stresses. $\mathrm{Zh}$ Prikl Mekh Tekhn Fiz, 2003;44(3):169-78.

44. Dunn JE, Fosdick RL. The morphology and stability of material phase. Arch Rat Mech Anal 1980;74(1):1-29.

45. Leibenson LS. Collected Papers. Vol. 4. Hydroaeromechanics. Geophysics. Moscow: Akad. Nauk SSSR; 1955.

46. Fu YB, Freidin AB. Characterization and stability of two-phase piecewise-homogeneous deformation. Proc Roy Soc London Ser A 2004;460:3065-94.

47. Kunin IA. Elastic Media with Microstructure. II. Three Dimensional Models. Berlin: Springer, 1983. 272 p.

48. Knowles JK. On the dissipation associated with equilibrium shocks in finite elasticity. J Elasticity 1979;9:131-58.

49. Lurie AI. Theory of Elasticity. Berlin: Springer; 2005.

50. Fosdick R, Royer-Carfagni GF. Alloy separation of a binary mixture in a stressed elastic sphere. J Elasticity 1996;42(1):49-77.

Translated by E.L.S. 